\title{
Ophthalmic Diagnostic Imaging: Glaucoma
}

\author{
Robert N. Weinreb, Christopher Bowd, \\ Sasan Moghimi, Ali Tafreshi, Sebastian Rausch, \\ and Linda M. Zangwill
}

\subsection{Introduction}

The detection and monitoring of glaucoma customarily involves several processes that include diagnostic modalities such as subjective evaluation of the optic nerve head $(\mathrm{ONH})$, visual field testing, and intraocular pressure measurements. These traditional methods of assessing glaucoma have several key limitations that dictate the need for supplementary approaches. The diagnostic assessment of the ONH in glaucoma by ophthalmoscopic examination or serial stereoscopic photographs is highly dependent on observer skills, inducing high inter-observer and intra-observer variation that affects its utility [1-3]. Visual field analysis through automated perimetry is a widely used technique that is considered an established clinical endpoint and is arguably the goldstandard for evaluation of glaucoma and for monitoring of disease progression. Although it is sensitive and specific at detecting glaucomatous functional loss, automated perimetry has several

R. N. Weinreb · C. Bowd · S. Moghimi · L. M. Zangwill Hamilton Glaucoma Center, Shiley Eye Institute, and The Viterbi Family Department of Ophthalmology, University of California San Diego, La Jolla, CA, USA

A. Tafreshi $(\bowtie) \cdot S$. Rausch

Heidelberg Engineering GmbH,

Heidelberg, Germany significant limitations [4-7]. The test requires the subjective input of the tested individual, making it prone to high short- and long-term fluctuation. The high fluctuation of the test, induced by its subjective nature, necessitates multiple tests to improve the reliability of the technique, delaying the recognition of glaucomatous damage [8]. Several studies have shown that detectable glaucomatous field abnormalities may be preceded by structural changes of the $\mathrm{ONH}$ and nerve fiber layer [9-18] Furthermore, intraocular pressure (IOP) is the major identified risk factor for the development of glaucomatous damage and is the only modifiable risk factor to date. Although lowering IOP serves to impede the progression of retinal ganglion cell degenerative change [1921], the high inter-individual variability and the diurnal variation in the intraocular pressure have limited the use of this parameter for the detection of the disease. Moreover, intraocular pressure values do not indicate whether damage has occurred, or to what extent. In addition, elevated IOP (i.e. ocular hypertension) does not necessarily result in glaucomatous damage [14].

While detection of glaucomatous structural damage to the eye during the earliest stage and precise assessment of this change are critical aspects of managing the disease, both feats are challenging. Glaucomatous damage is largely irreversible and, therefore, eyes with structural damage must be identified as early and as 
accurately as possible because they are at risk for continued injury. It has been suggested that the earlier glaucoma is detected and treated, the greater the likelihood that medical or surgical intervention will delay or prevent the progression of glaucomatous neuropathy and subsequent functional impairment [22-24]. Furthermore, because glaucoma progresses slowly, it is important to detect real change due to disease that is beyond normal age-related loss and short-term and long-term fluctuations. This assumption underscores the need for an accurate and reproducible quantitative evaluation of the eye.

During the past three decades, there has been significant development and implementation of several imaging technologies designed to objectively and quantitatively detect glaucomatous neuropathy at early stages of disease. Beyond early detection, quantitative and objective imaging devices offer a more sensitive way to detect glaucomatous progression when compared with clinical qualitative assessments. One of the earliest imaging devices introduced to the ophthalmic field was a confocal scanning laser ophthalmoscope (cSLO), a device developed to assess optic disc topography in the late 1980s (Laser Tomographic Scanner, Heidelberg Instruments). With reductions in cost and the advent of improved hardware, the introduction of the first practical commercial cSLO device was introduced in 1991 [Heidelberg Retina Tomograph (HRT); Heidelberg Engineering, Heidelberg, Germany].

Imaging instruments provide objective, quantitative measures of neuroretinal rim thinning, RNFL atrophy, and excavation of the optic cup, and are increasingly utilized in the clinical management of glaucoma patients. This is due in part to the availability of summary information that can easily be used in clinical management decisions. For example, most instruments now include a reference database for making statistical indications of whether a patient measurement is "Within Normal Limits," "Borderline," or "Outside Normal Limits." In addition, each device provides a measure of image quality so that the clinician can determine whether the image is of sufficient quality to be utilized in clinical management decisions. With continuous developments in imaging technology like spectral domain OCT (SD-OCT) and advancements in research applications of such technologies, the value of these devices in glaucoma management is likely to continue growing.

Although in vivo imaging with cSLO, timedomain OCT (TD-OCT), and spectral-domain OCT (SD-OCT) have been commercially available for the management of glaucoma for over 10 years, interpretation and utilization of such results remains a challenge. However, clinical research continues to significantly advance the relevance and utility of diagnostic imaging devices by enabling visualization of highresolution and detailed images and to provide sophisticated data analysis strategies. Such improvements increase efficiency while providing precise, accurate analysis of the retinal data produced by each device [25-27]. This chapter will review diagnostic imaging techniques that have advanced and continue to advance diagnosis and management of glaucoma.

\subsection{The Heidelberg Retina Tomograph: Confocal Scanning Laser Ophthalmoscope (cSLO)}

The first cSLO device developed as a diagnostic aid for glaucoma, the Heidelberg Retina Tomograph (HRT), utilizes confocal optics to obtain multiple measures of retinal height at consecutive focal planes to provide a topographic map that extends from the retinal anterior surface down to the lamina cribrosa. The HRT platform includes comprehensive software that facilitates image acquisition, storage, retrieval, and analysis. After manually delineating the optic disc margin by placing a contour line along the inner edge of the scleral ring at the baseline exam, stereometric parameters are provided to describe the retinal topography of each image. The contour line is automatically transferred to all follow-up examinations. Many of the stereometric parameters are calculated based on a standard reference plane set $50 \mu \mathrm{m}$ posterior to the average contour 
line height (i.e., retinal height) at a $5^{\circ}$ sector along the temporal rim; an area thought to be least effected by glaucomatous progression and therefore thought to change minimally over time. Stereometric parameters include: disc area (area within contour), rim area (area within contour and above reference plane), cup area (area within contour and below reference plane), rim volume, cup volume, mean cup depth, mean height of contour, an indirect measure of retinal nerve fiber layer (RNFL) thickness, and cup shape (Fig. 5.1).

\subsubsection{Clinical Development}

Several key clinical research studies contributed to the advancement and development of the cSLO technology for assessment of the optic nerve head. In 1993, Weinreb et al. recommended acquiring multiple images at each visit, and showed that with three images one could obtain excellent reproducibility within a short time for a reasonable cost [28]. This study led to the implementation of software that automatically acquires three sets of three-dimensional images each time. If the quality of images in at least one of the series is not good enough (for reasons such as fixation loss), the acquisition is automatically continued until three useful series are obtained. The same group also showed the SLO imaging is highly reproducible in patients with [29] or without undilated pupils [30]. There was no significant difference between the standard deviation of a single height measurement in normal and glaucomatous eyes. No correlation was found between standard deviation of the measurements and pupil size or age of the subject [29]. Zangwill et al. demonstrated a moderate agreement between clinicians and a HRT in estimating cup/disc ratios with the highest disagreements in discs with gradual slopes and pallor. New quantitative criteria then were established for characterizing a disc as glaucomatous using HRT [31]. A quantitative method was then developed for analysis of the topographic relationship between structural and functional damage in patients with glaucoma [32].

In 1998, Wollstein and associates introduced the Moorfields Regression Analysis (MRA) to the
HRT. The MRA classification technique compares global and local rim area measurements (reference plane dependent) to a normative database taking into account disc area and age [33]. A few years later, a machine-learning based diagnostic classifier called the Glaucoma Probability Score (GPS) was introduced and implemented in the third generation of the HRT (HRT III). The GPS was one of the earliest applications of machine learning in the ophthalmic field, using a geometric model to describe the shape of the optic disc/parapapillary retina (globally and locally) based on five parameters (cup size, cup depth, rim steepness, horizontal retinal nerve fiber layer curvature, and vertical retina nerve fiber layer curvature) [34]. These parameters are then interpreted by a relevance vector machine classifier [35] and the resulting output describes the probability that the eye is glaucomatous (based on fit to training data from healthy and glaucoma eyes). This technique does not depend on an operator drawn contour line or a reference plane and is therefore operator independent (Fig. 5.2). Results from both classification techniques are reported as 'within normal limits', 'borderline' or 'outside normal limits' globally, and for each of six disc sectors relative to the normative data.

In 2000, Chauhan et al. [36] introduced Topographic Change Analysis (TCA), a progression analysis tool that became a gold-standard in the assessment of glaucomatous ONH changes. The TCA quantifies the change in the topography of the ONH using the first image as the baseline and the subsequent images as follow-up examinations. The TCA does not require a defined contour line to determine areas of significant change as it assesses the height of the optic disc and retinal surface at each follow-up measurement and compares these to the baseline measurement. The images are further analyzed using an array of $4 \times 4$ pixels, called super-pixels. Superpixels allow for pooling over a larger area and yield more repeated measures for analysis. In steep areas like the edge of the cup, the variability is greater than that in flat areas. The topographic measurement of a superpixel $(4 \times 4$ pixels $)$ and an analysis of variance model for each superpixel is then calculated. 
Heidelberg Retina Tomograph OU Report

HEIDELEETG EПБIПEЕГIПG

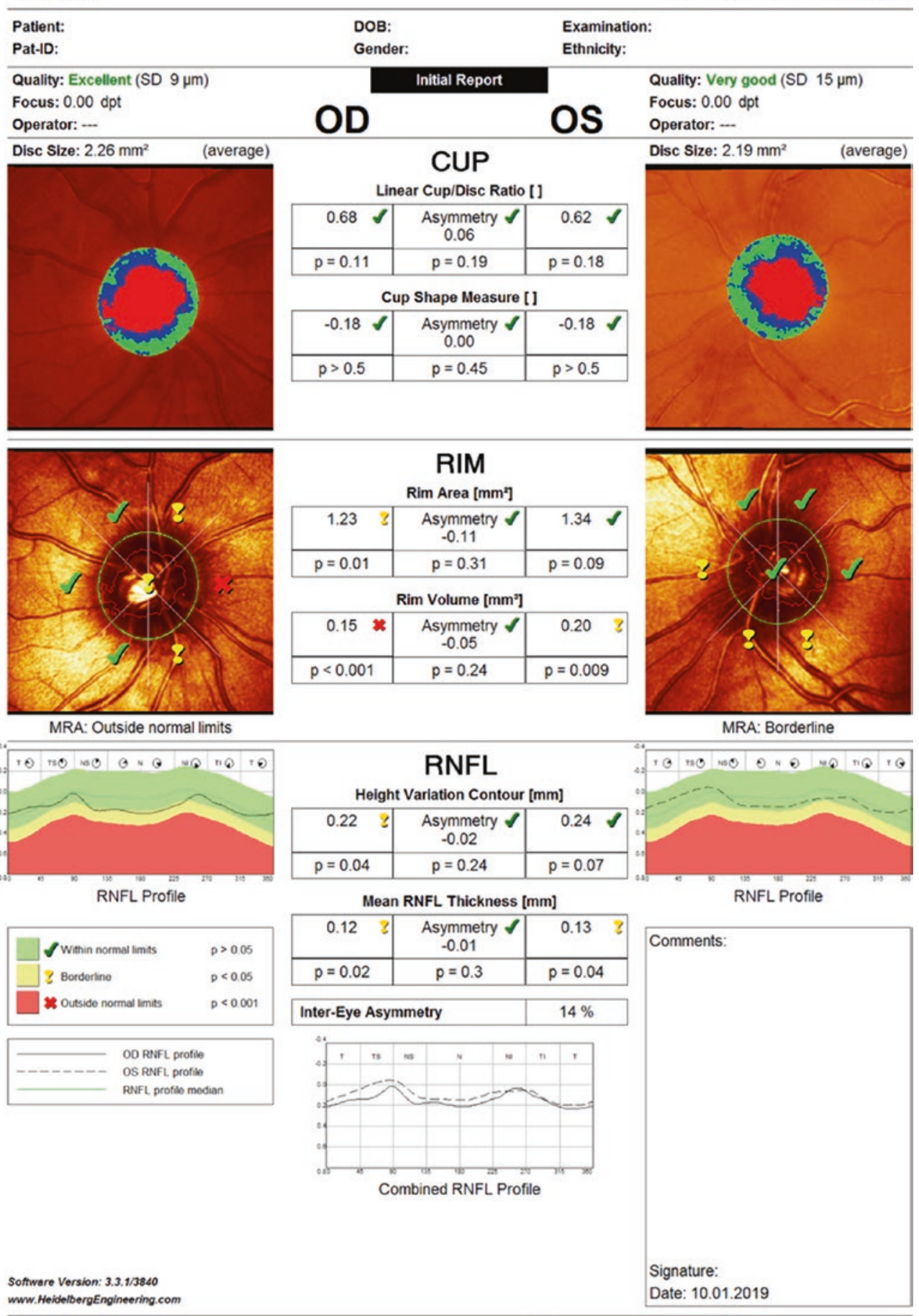

Fig. 5.1 After manually delineating the optic disc margin by placing a contour line along the inner edge of the scleral ring at the baseline exam, stereometric parameters are provided to describe the retinal topography of each image. Stereometric parameters include: disc area (area within contour), rim area (area within contour and above reference plane), cup area (area within contour and below reference plane), rim volume, cup volume, mean cup depth, mean height of contour, an indirect measure of retinal nerve fiber layer (RNFL) thickness, and cup shape 
Heidelberg Retina Tomograph GPS Report

매티트륻드

\begin{tabular}{|c|c|c|}
\hline Patient: & DOB: & Examination: \\
\hline Pat-ID: & Gender: & Ethnicity: \\
\hline Quality: Very good (SD $15 \mu \mathrm{m}$ ) & Follow-Up Report & Quality: Very good (SD $13 \mu \mathrm{m}$ ) \\
\hline Focus: $3.00 \mathrm{dpt}$ & & Focus: $3.00 \mathrm{dpt}(\mathrm{AL}-0.5)$ \\
\hline Operator: DA & & Operator: DA \\
\hline
\end{tabular}
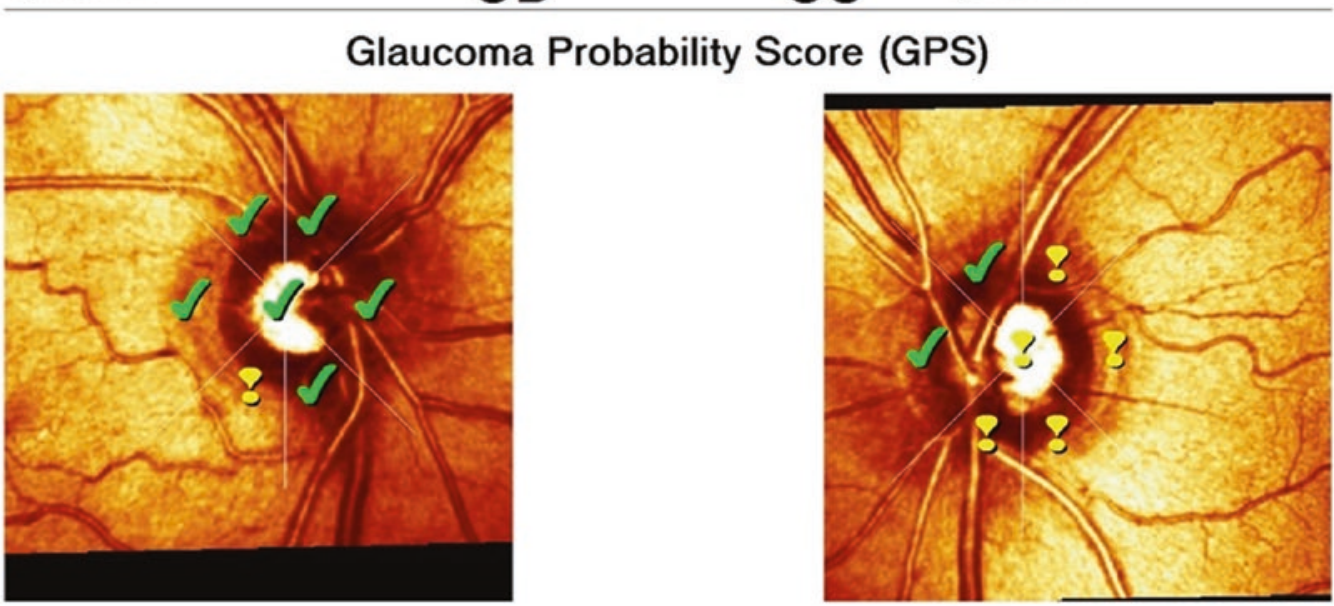

\begin{tabular}{|c|c|c|c|c|c|c|c|c|c|c|c|c|c|c|}
\hline onoter & tempoceat & Imosup & molint & ansat & ondsoos & astint & Parameter & onos & nempecal & tmovero & implint & nosat & nutros & sulint \\
\hline a.28 & 023 & 025 & 028 & 024 & 0.25 & 027 & Glouccema percel. & 0.90 & 0.20 & 0.31 & 0.94 & 028 & $02 a$ & 028 \\
\hline ses & 240 & -974 & $\$ 67$ & - 985 & $9 n$ & $\rightarrow 90$ & Rm steesoess & 964 & -0.53 & oes & -9.90 & -876 & 0.32 & $\$ 900$ \\
\hline on & - & - & - & - & - & - & Cop deparimming & on & - & - & - & - & - & - \\
\hline 001 & - & - & - & - & - & - & H. RESL cun. & $\$ 02$ & - & - & - & - & - & - \\
\hline s.16 & - & - & - & - & - & - & V. Rost cun. & 0.15 & - & - & - & - & - & - \\
\hline
\end{tabular}

Outside normal limits

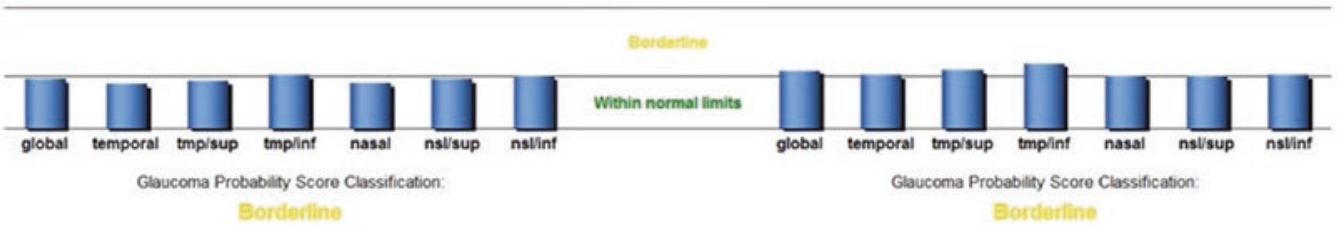

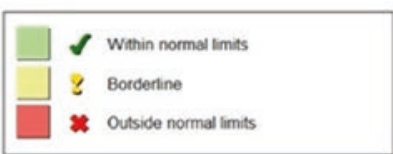

Software Version: 3.3.1/370 www. Heide/bergEngineering.com

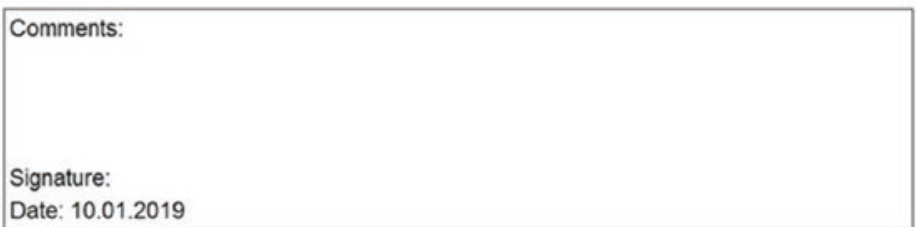

depend on an operator drawn contour line or a reference plane and is therefore operator independent. Results are reported as 'within normal limits', 'borderline' or 'outside normal limits' globally, and for each of six disc sectors relative to the normative data
Fig. 5.2 The Glaucoma Probability Score (GPS) uses a geometric model to describe the shape of the optic disc/ parapapillary retina (globally and locally) based on five parameters. The resulting output describes the probability that the eye is glaucomatous. This technique does not 


\subsubsection{Clinical Validation}

The combination of these HRT (cSLO) diagnostic tools became the gold standard for imaging and monitoring of the optic nerve head in glaucoma, in the early- to mid-2000s. Several large and seminal studies contributed to the validation of the diagnostic parameters offered by the HRT.

Bowd et al. validated the clinical utility of this tool by showing that TCA parameters can discriminate between progressing glaucoma eyes and longitudinally observed healthy eyes, suggestive of the ability of the HRT with TCA to detect early ONH changes due to glaucoma [37]. The Ocular Hypertension Treatment Study (OHTS), sponsored by the National Institutes of Health/ National Eye Institute, is a multicenter randomized clinical trial designed to evaluate the safety and efficacy of topical glaucoma medication in delaying or preventing the onset of glaucomatous VF loss or optic nerve deterioration in participants with ocular hypertension [14, 38]. In OHTS, the presence of clinically significant disc changes during follow-up was determined by evaluation of serial stereoscopic optic disc photographs.

The Confocal Scanning Laser Ophthalmoscopy Ancillary Study to the Ocular Hypertension Treatment Study was the first multicenter clinical trial to use cSLO imaging to monitor changes in the optic disc [39]. This study evaluated the effectiveness of various HRT parameters in detecting the presence and progression of glaucomatous optic disc damage and determined whether optic disc topographic measurements are an accurate predictor of visual field loss. The 451 participants in the OHTS CSLO ancillary study were recruited from seven of the 22 OHTS centers and the HRT examinations were obtained annually after pupillary dilation at the time of scheduled OHTS fundus examination and optic disc photography.

The baseline data from OHTS indicated that cSLO measurements correlated well with expert evaluation of stereoscopic photography and that differences in topographic optic disc parameters between African Americans with ocular hypertension and other racial groups are largely explained by the larger optic disc area in the African
Americans [38-40]. This latter result highlighted the need to consider race and optic disc size when evaluating the appearance of the optic disc in glaucoma. Therefore, several of the parameters implemented in the HRT are reported and compared to a race specific normative database.

\subsubsection{Surrogate Endpoints and Progression}

The HRT neuroretinal rim parameters have been shown to be predictive of functional loss and to serve as suitable surrogate endpoints in glaucoma clinical trials [15, 41-44]. The OHTS data also showed that baseline topographic optic disc measurements can predict the onset of primary open angle glaucoma in patients with ocular hypertension [15]. OHTS results suggest that baseline GPS, MRA, and stereometric parameters alone or when combined with baseline clinical and demographic factors can be used to predict the development of POAG endpoints in OHTS participants and are as effective as stereophotographs for estimating the risk of developing POAG in ocular hypertensive subjects [15, 41]. In 2008, Alencar et al. assessed whether baseline GPS results are predictive of progression in patients suspected of having glaucoma. Their study results showed that baseline GPS are in fact predictive and that they perform as well as subjective but expert assessment of the optic disc. They further suggested that the HRT GPS could potentially replace stereophotographs as a tool for estimating the likelihood of conversion to glaucoma [42]. In 2009, Chauhan et al. concluded that patients that presented with glaucomatous visual field progression were up to three times more likely to have prior disc changes as measured on TCA [43]. Medeiros et al., showed that progressive rim area loss, as defined by the HRT parameters, was highly predictive of the development of visual field loss in glaucoma and explained a significant proportion of the effect of treatment on the clinically relevant outcome [44]. They suggested that rim area measurements may be suitable surrogate endpoints in glaucoma clinical trials [44]. 
The Diagnostic Innovations in Glaucoma Study (DIGS) and the African Descent and Glaucoma Evaluation Study (ADAGES) are large multi-center ongoing studies that include normal subjects, patients with glaucoma, and glaucoma suspects, who are semi-annually evaluated clinically and with several functional and optical imaging tests including HRT and SD-OCT. The 3-site collaboration includes the Hamilton Glaucoma Center at the Veterbi Family Department of Ophthalmology (RN Weinreb), University of California, San Diego (UCSD) (Data Coordinating Center) (L Zangwill), Columbia University and the New York Eye and Ear Infirmary (J Liebmann), and the Department of Ophthalmology, University of Alabama, Birmingham (C Girkin and M Fazio) [45, 46].

Results from DIGS confirmed earlier reports of the comparability of stereophotograph based cup-to-disc ratio measurements and HRT measures in predictive models [47]. The same group also concluded that the presence of optic disc damage on stereophotographs is highly predictive of future development of functional loss [48]. They later showed that the rate of rim area loss measured using HRT is approximately 5 times faster in eyes in which POAG developed compared with eyes in which it did not. The results of this study, suggest that measuring the rate of structural ONH change using cSLO-based parameters can provide important information for the clinical management of ocular hypertensive patients [49].

The ADAGES group characterized the rate and pattern of age-related and glaucomatous neuroretinal rim area changes in subjects of African and European descent, using HRT parameters. They showed that compared with healthy eyes, the mean rate of global rim area loss was 3.7 times faster and the mean rate of global percentage rim area loss was 5.4 times faster in progressing glaucoma eyes [50].

\subsubsection{Summary}

Heidelberg Retina Tomograph cSLO technology introduced the ability to objectively quantify var- ious diagnostic parameters for the assessment and management of glaucoma. The technology's diagnostic parameters offer clinicians with an objective and precise method to aid their decision in the diagnosis and management of the disease, while also serving as potential surrogate endpoints in clinical trials. A more recent imaging technology, spectral domain optical coherence tomography (SD-OCT), which also enables to objectively image and assess the optic nerve head as well as the retinal nerve fiber layer and macula, has been established as the most current commonly used diagnostic imaging aid for glaucoma.

\subsection{SPECTRALIS SD-OCT}

With the introduction of SD-OCT, it has become possible to image ocular structures in three dimensions with high axial resolution, fast scan rates, and high contrast. These advances have improved visualization of small details and provided a platform for precise analytics. The benefits of SD-OCT technology, combined with eye-tracking algorithms that enable precise scan registration from session to session allow for reliable removal of errors induced by eye movements. The resulting scans offer detailed visualization of retinal structures and provide accurate segmentation of the anatomical boundaries used in advanced analytics.

The SPECTRALIS SD-OCT (Heidelberg Engineering $\mathrm{GmbH}$, Heidelberg, Germany) incorporates a real-time eye tracking system (TruTrack ${ }^{\mathrm{TM}}$ ) that couples cSLO and SD-OCT scanners to adjust for eye movements and to ensure that the same precise location of the retina is scanned time after time (Fig. 5.3) reducing variability across longitudinal measurements used for monitoring disease progression. This method also allows B-Scans to be re-sampled in the same location to improve the signal-to-noise ratio (SNR), a technique called Automatic Realtime Tracking (ART).

The standard SPECTRALIS SD-OCT glaucoma software includes RNFL thickness measurements derived from a $12^{\circ}$ circle scan, 


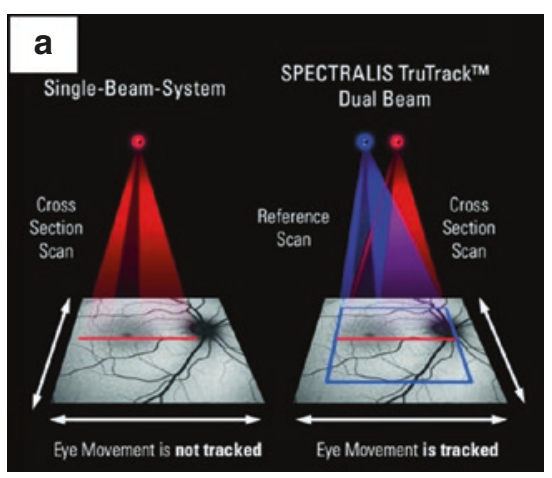

Fig. 5.3 A core technology of the SPECTRALIS is TruTrack (a), a dual-beam tracking system which provides very important clinical benefits such as retinal recognition, follow-up scanning, precise co-localization of

manually centered by the operator on the optic disc. The RNFL thickness analysis then provides sectorial and global measurements that are compared with a reference database of healthy controls. The RNFL thickness values for a scan are classified using a 6-sector analysis, and sectors can be flagged green ("Within Normal Limits"), yellow ("Borderline"), and red ("Outside Normal Limits"). RNFL parameters of the SPECTRALIS have been evaluated relative to other commercially available SD-OCT devices and shown to be as specific, sensitive, and repeatable [51, 52].

While several SD-OCT devices are commercially available, Heidelberg Engineering has included several unique features and functionalities with the intent to account for relevant factors that may influence the resulting diagnostic parameters. The Glaucoma Module Premium Edition (GMPE) offers new scan patterns and an updated reference database that was acquired using these new features and functionalities, designed to enhance and support the clinical assessment of glaucoma by accounting for anatomic variability of each eye to improve classifications.

\subsubsection{Clinical Assessment of Optic Nerve Head Parameters}

The retinal ganglion cell (RGC) axons comprise the RNFL with axons exiting the eye via the optic fundus images with depth-resolved information in OCTscans. This system also enables the Automatic Real-time Tracking (ART) technique for image averaging to improve image quality by reducing noise (b)

nerve head. The health of the optic nerve head can be assessed based on the amount of neuroretinal rim tissue present. Because the axons exiting the eye make up a significant portion of the neuroretinal rim, its loss is associated with RGC and axonal degeneration, both of which are characteristic of glaucomatous damage. The optic disc constitutes the clinically visible surface of the neural and connective tissues of the ONH. The health of the neuroretinal rim is defined using two landmarks: the optic disc margin and the optic cup margin. These two landmarks define the outer edge (clinical disc margin) and inner edge (optic cup margin) of the neuroretinal rim. The amount of rim tissue then is estimated within the apparent plane of the disc margin as either the ratio of the size of the cup to the size of the disc [53] or the rim area [54]. An eye that exhibits a large CDR is indicative of potential glaucomatous damage as axonal loss results in expansion of the optic disc cup. However, both the optic disc and cup margin are defined subjectively and are difficult to delineate consistently [55]. Therefore, the resultant CDR and clinical neuroretinal rim quantification are variable. Furthermore, these concepts are applied whether the examination is performed with direct ophthalmoscopy, slit-lamp biomicroscopy, optic disc photography, or a number of quantitative imaging methods.

The more recent SPECTRALIS SD-OCT with OCT2 Module technology provides a new level 
of high-resolution imaging of $\mathrm{ONH}$ anatomic features that are affected in glaucoma. Clinicians can now visualize optic nerve structures such as the anterior and posterior lamina cribrosa surfaces, Bruch's membrane-retinal pigment epithelium complex and its termination within the $\mathrm{ONH}$ border, tissue of Elschnig, and the scleral canal opening [55-65]. Studies show that accurate colocalization of fundus photographs to SD-OCT image data allows clinicians to identify structures that correspond to common clinical landmarks such as the optic disc margin [56, 66]. SPECTRALIS imaging and measurement of the ONH landmarks support interpretation of fundus images and can objectively assist in clinical assessment of the nerve in three dimensions.

\subsubsection{Bruch's Membrane Opening (BMO) in SD-OCT-Based Neuroretinal Rim Measurements}

More recent SD-OCT imaging studies have challenged concepts of the clinical disc margin and rim quantification from both anatomic [55] and geometric [66-68] perspectives. The termination of Bruch's membrane at the $\mathrm{ONH}$ marks the opening through which retinal ganglion cell axons exit the eye to form the choroidal and scleral portions of the neural canal. Because axons cannot pass through an intact Bruch's membrane to exit the eye, this anatomic opening, termed Bruch's membrane opening (BMO), is a true anatomical border of the neural tissue. Thus, the BMO is a stable anatomical landmark from which neuroretinal rim measurements can be made (Fig. 5.4) [69].

However, Bruch's membrane is a very thin anatomical structure that is about $2-5 \mu \mathrm{m}$ thick, and it appears as a hyper-reflective layer on SD-OCT that is approximately as thick as the RPE, around 14-16 $\mu \mathrm{m}$ [70]. High-resolution and excellent signal-to-noise ratio (SNR) SD-OCT images are needed in order to detect the reflectance of bounding surfaces. It has been shown that the SPECTRALIS SD-OCT can consistently identify the BMO and that these images correlate to ground-truth $\mathrm{ONH}$ histology, as

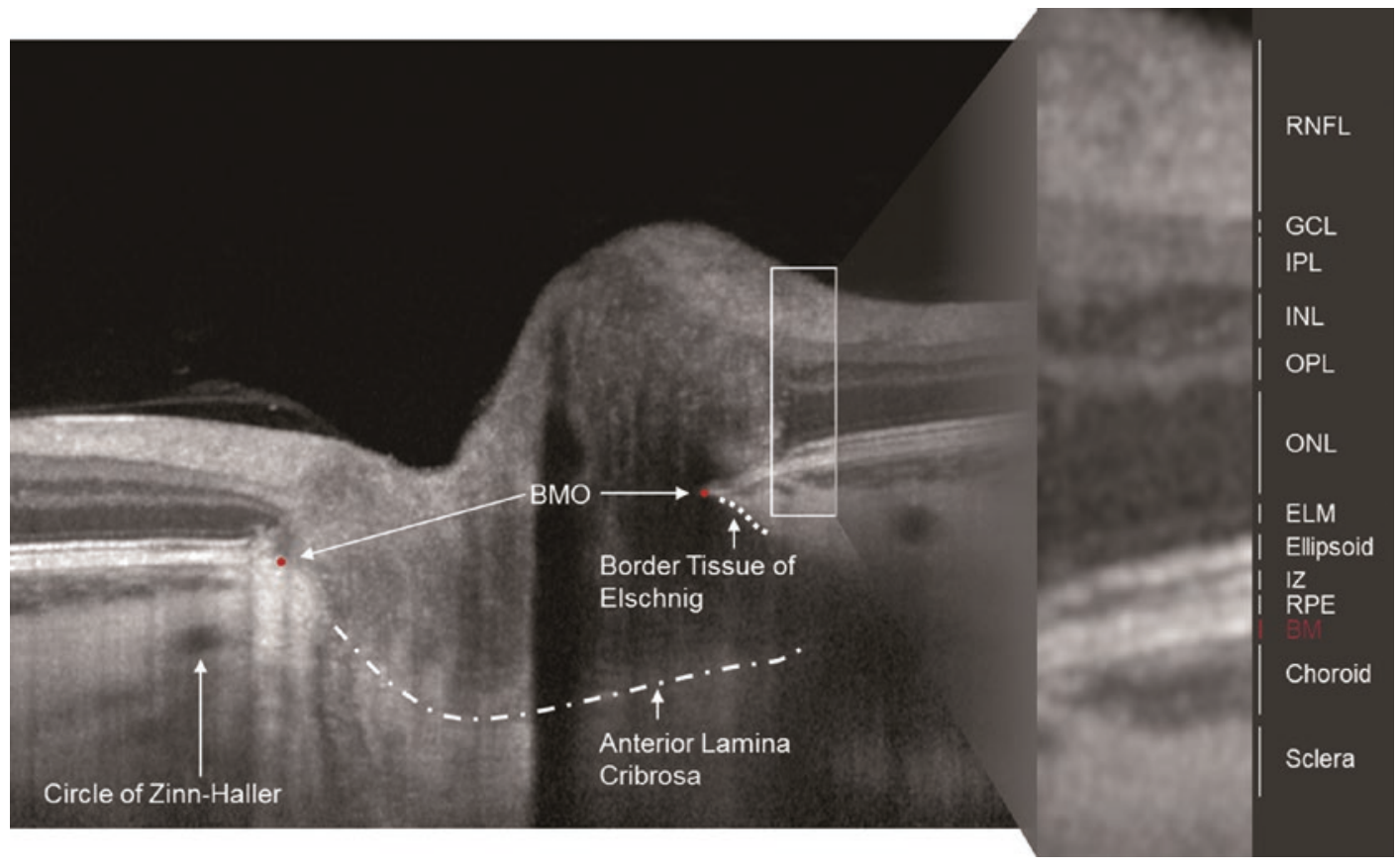

Fig. 5.4 The BMO represents a stable structure through which all axons exit the eye. Because blood vessels and axons cannot pass through Bruch's membrane, it is considered an appropriate anatomical boundary of the optic disc 

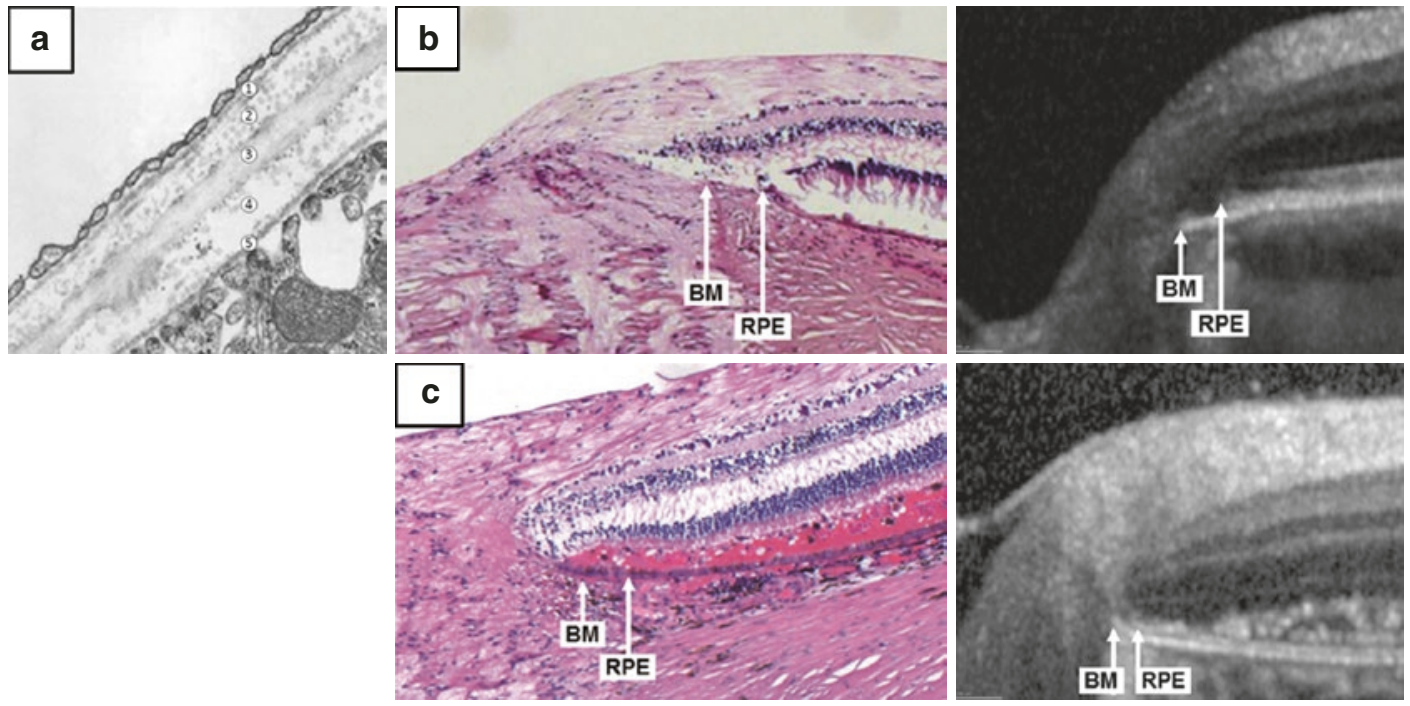

Fig. 5.5 (a) Electron microscopy of Bruch's membrane shows the basal membrane of the choriocapillaris, collagen layers, elastic layer, and basal membrane of the
RPE. Panels (b) and (c) show ONH histology of Bruch's membrane, along with its corresponding appearance on SD-OCT. Images courtesy Christian Mardin, MD shown in Fig. 5.5 [56, 66, 71]. Finally, the stability of BMO under a variety of conditions provides another rationale for its usefulness as a landmark over time [69]. BMO also is unaltered by large changes in IOP induced by glaucoma surgery, as the two-dimensional plane that best fits BMO is axially stable with surgical reduction of IOP [72].

The orientation of the neuroretinal rim relative to the $\mathrm{BMO}$ varies around the $\mathrm{ONH}$ because axons can exit the eye along varying paths, ranging from parallel to the visual axis to perpendicular to it [73]. In order to correctly account for these variations, studies have demonstrated that the minimum distance from BMO to the internal limiting membrane represents the most geometrically accurate measurement of neuroretinal rim width [66-68]. This neuroretinal rim measurement has been termed Bruch's Membrane Opening-Minimum Rim Width (BMO-MRW). Studies have demonstrated the usefulness of BMO-MRW in the detection of progressive ONH change in experimental animal models of glaucoma and in human eyes [69, 74]. The BMOMRW is diagnostically specific and sensitive for detecting glaucoma, and it enhances the clinical assessment of the optic disc [75]. The BMO-MRW parameter provides better diagnostic performance than the original gold-standard HRT analyses [76]. Ultimately, the BMO-MRW and RNFLT measurements have been shown to complement each other in the assessment and monitoring of glaucoma [77].

The SPECTRALIS GMPE optic nerve headradial and circle (ONH-RC) scan acquires 24 radial and three concentric circle scans, with diameters of $3.5,4.1$ and $4.7 \mathrm{~mm}$, centered on the BMO (Fig. 5.6). The radial scans define 48 BMO points that serve as the basis for the BMOMRW measurements, and the three circumpapillary RNFL scans offer complementary RNFL thickness measurements that capture valuable information away from the optic nerve head. The BMO-MRW measurements of the radial scans and the RNFL measurements of the three circle scans are both adjusted for BMO area and age. The circle scans show comparable diagnostic performance, and in patients with large areas of peripapillary atrophy, the outer scans may offer reliable RNFL measurements when the conventional $3.5 \mathrm{~mm}$ scan is confounded by atrophy [78]. 

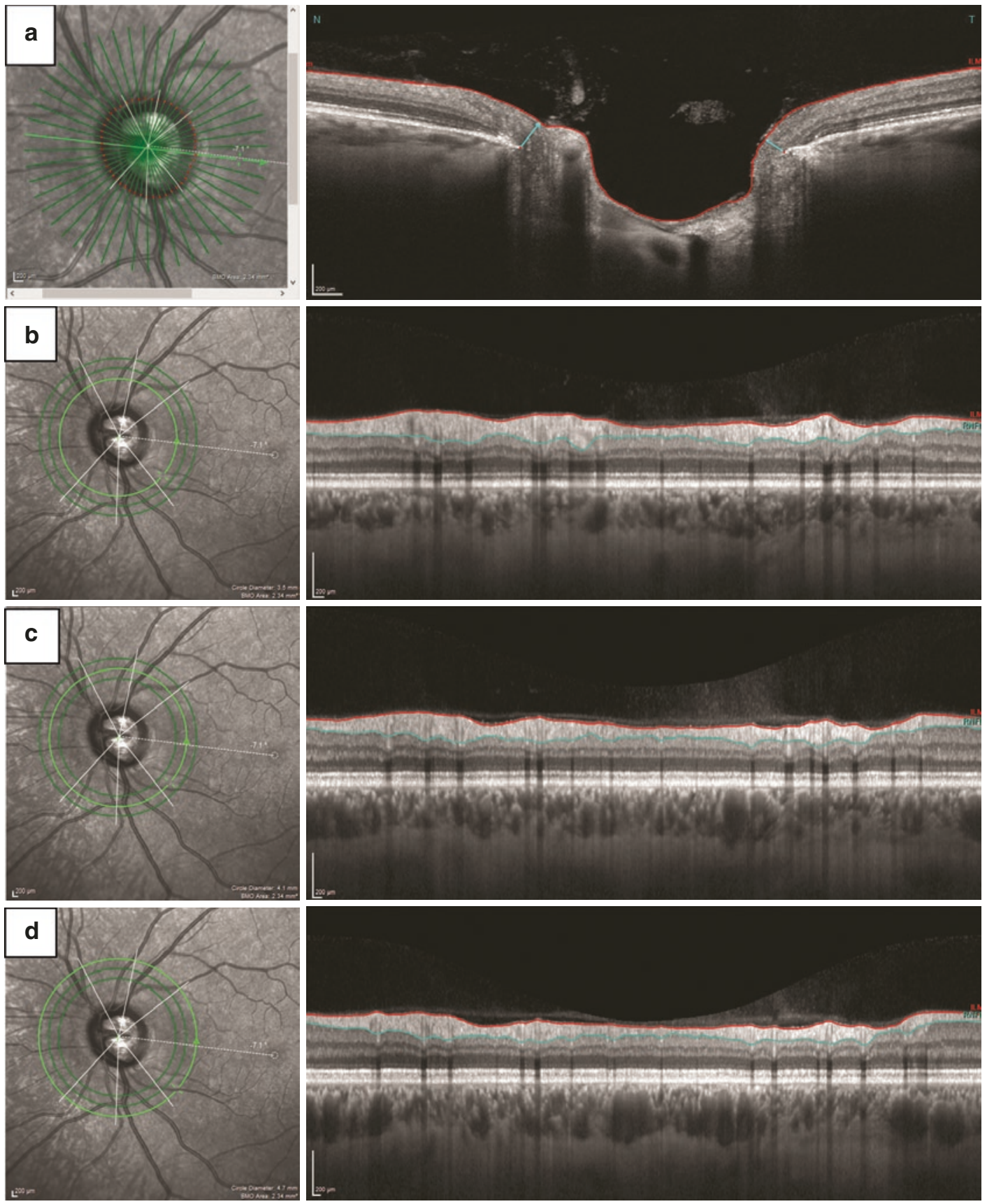

Fig. 5.6 The ONH-RC scan pattern produces 24 line scans and 48 BMO endpoints, shown in (a). The three often offer complementary information and can confirm the presence of focal RNFL wedge defects that broaden concentric circumpapillary scans have a diameter of $3.5 \mathrm{~mm}(\mathbf{b}), 4.1 \mathrm{~mm}(\mathbf{c})$, and $4.7 \mathrm{~mm}(\mathbf{d})$. The three scans away from the ONH (white arrows). Images courtesy Maria Pilar Bambó, MD, PhD 


\subsubsection{Anatomic Variation: Position of the Fovea Relative to the Center of the ONH}

On average, the fovea is located $7^{\circ}$ below the level of the center of the $\mathrm{ONH}$, but the angle can vary from $6^{\circ}$ above to $29^{\circ}$ below [79]. Although the positions of the fovea and $\mathrm{ONH}$ center vary considerably between subjects, the anatomic path of RNFL bundles is governed primarily by these two structures as the bundles approach the $\mathrm{ONH}$ and exit the eye $[80,81]$. In fundus images, the positions of the fovea and $\mathrm{ONH}$ also may vary slightly within the same individual from day to day because of cyclotorsion [82], but the path of RNFL bundles remains constant relative to the fovea-BMO center (FoBMOC) axis as shown in Fig. 5.7 [81].

The FoBMOC axis could significantly vary between the two eyes of one individual. A clinical example of this disparity is shown in Fig. 5.8. If these variations are not taken into account, it may lead to artificially large inter-individual differences in sectoral measurements, reducing the diagnostic precision of the device. Errors in mapping ocular structures to the visual field also may be induced that could contribute to the somewhat poor correlation between measures of structure and function observed in glaucoma [83, 84].

To account for these issues, the SPECTRALIS GMPE offers a proprietary feature called the "Anatomic Positioning System" (APS). Image acquisition using the APS ensures that OCT images are acquired at fixed and known retinal locations relative to certain anatomical landmarks: the center of the fovea and the center of Bruch's membrane opening. The process of defining the APS landmarks is semi-automated within data acquisition, and the operator is able to adjust and confirm the landmarks that the device detects (Fig. 5.9). All subsequent GMPE scans are aligned to the baseline landmarks and are automatically oriented according to the patient's FoBMOC axis. FoBMOC-aligned scans ensure all eyes are anatomically aligned correctly and compared with healthy control eyes regardless of anatomical differences, thereby improving accuracy of the sector analysis (Fig. 5.8).

While the APS landmarks serve as the basis for accurate baseline measures of each individual eye, the previously mentioned TruTrack eyetracking feature accounts for changes in head position and ensures precise placement of follow up scans, both important for maintaining accurate

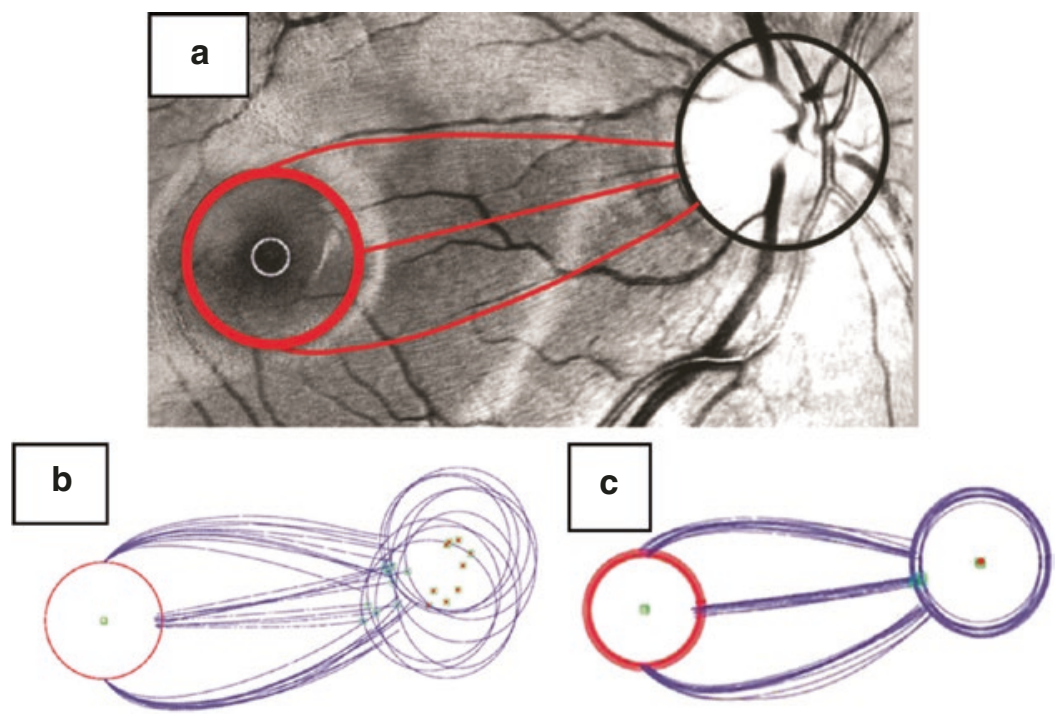

Fig. 5.7 Even as the angle of the fovea to the center of the $\mathrm{ONH}$ changes due to cyclotorsion or anatomical differences, the arcuate path of the RNFL bundles remains constant to this axis. This can be seen from the tracing of the RNFL fibers from the nerve to the fovea in (a). For 11 different eyes, the optic nerve position varies relative to the fovea as shown in (b), but alignment of the images in (c) illustrates the consistent path of the RNFL bundles. Figure 15 of [81], reprinted with permission 

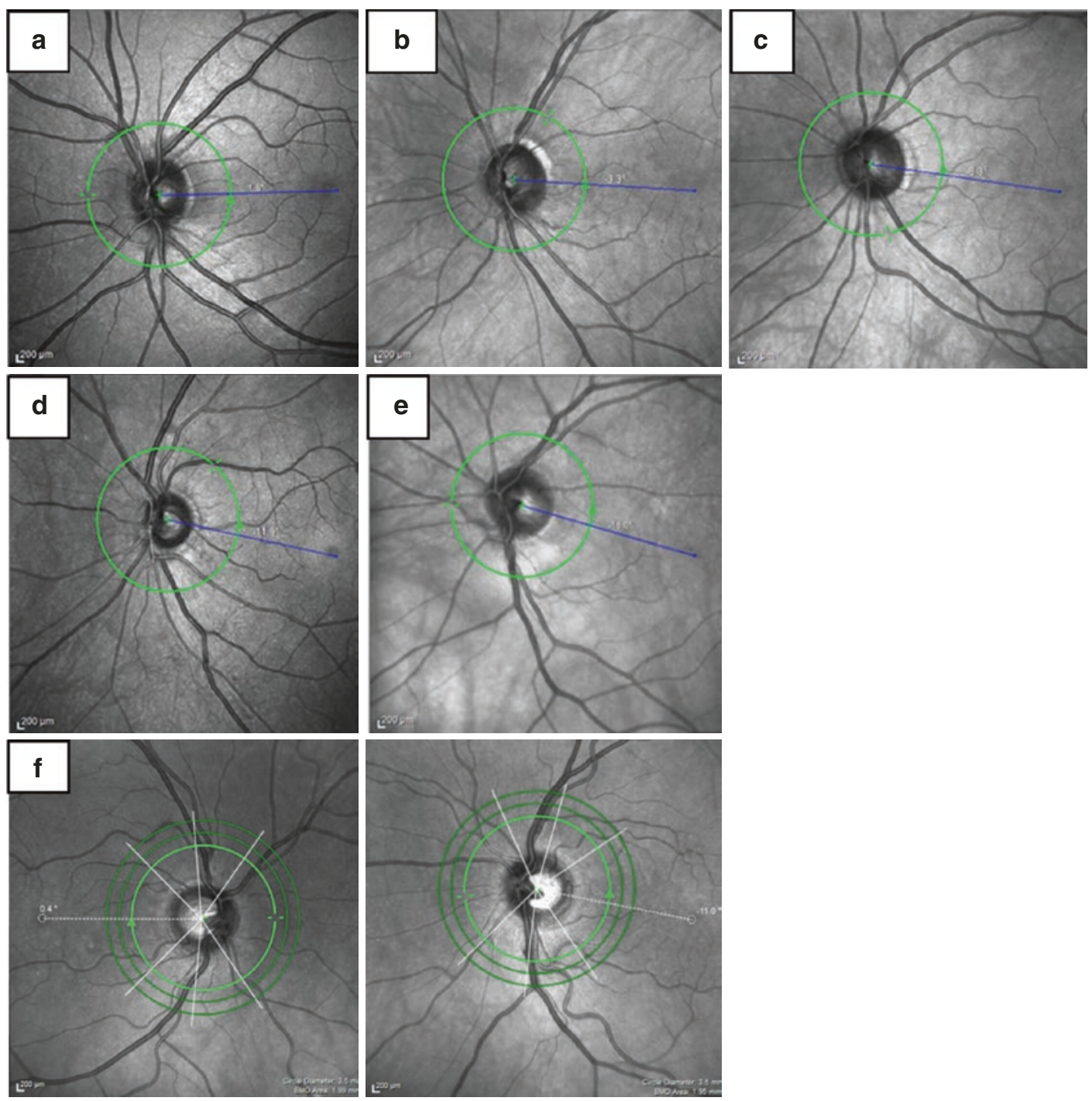

Fig. 5.8 The range of angles for the fovea to center of $\mathrm{ONH}$ axis is large across a population. In these five examples, the fovea is $+1.5^{\circ}$ in (a), $-3.3^{\circ}$ in $(\mathbf{b}),-8.3^{\circ}$ in $(\mathbf{c})$, $-11.9^{\circ}$ in $(\mathbf{d})$, and $-16.0^{\circ}$ in (e). Variability can also occur between the two eyes of a single patient, as seen in (f) with an angle of $+0.4^{\circ} \mathrm{OD}$ and $-11.0^{\circ} \mathrm{OS}$. The three white lines through the $\mathrm{ONH}$ in (f) represent the 6-sector Garway-Heath classification regions and are shifted according to the angle comparison to the reference database and when assessing progression [85].

\subsubsection{Anatomic Variation: ONH size and Ocular Magnification Impact RNFL Measurements}

In 1996, Schuman et al. reported that a circle diameter of $3.4 \mathrm{~mm}$ was the most accurate and reproducible scan size for RNFL thickness measurements [86]. Since then, most OCT instruments and studies have used circular scans with a diameter very close to $3.4 \mathrm{~mm}$, independent of $\mathrm{ONH}$ size. However, it is now generally recognized that the optic disc size shows a high interindividual variability, with areas ranging between 0.8 and $6.00 \mathrm{~mm}^{2}$ in normal eyes [87]. Histological studies have shown that RNFL thickness decreases with increasing distance from the optic 

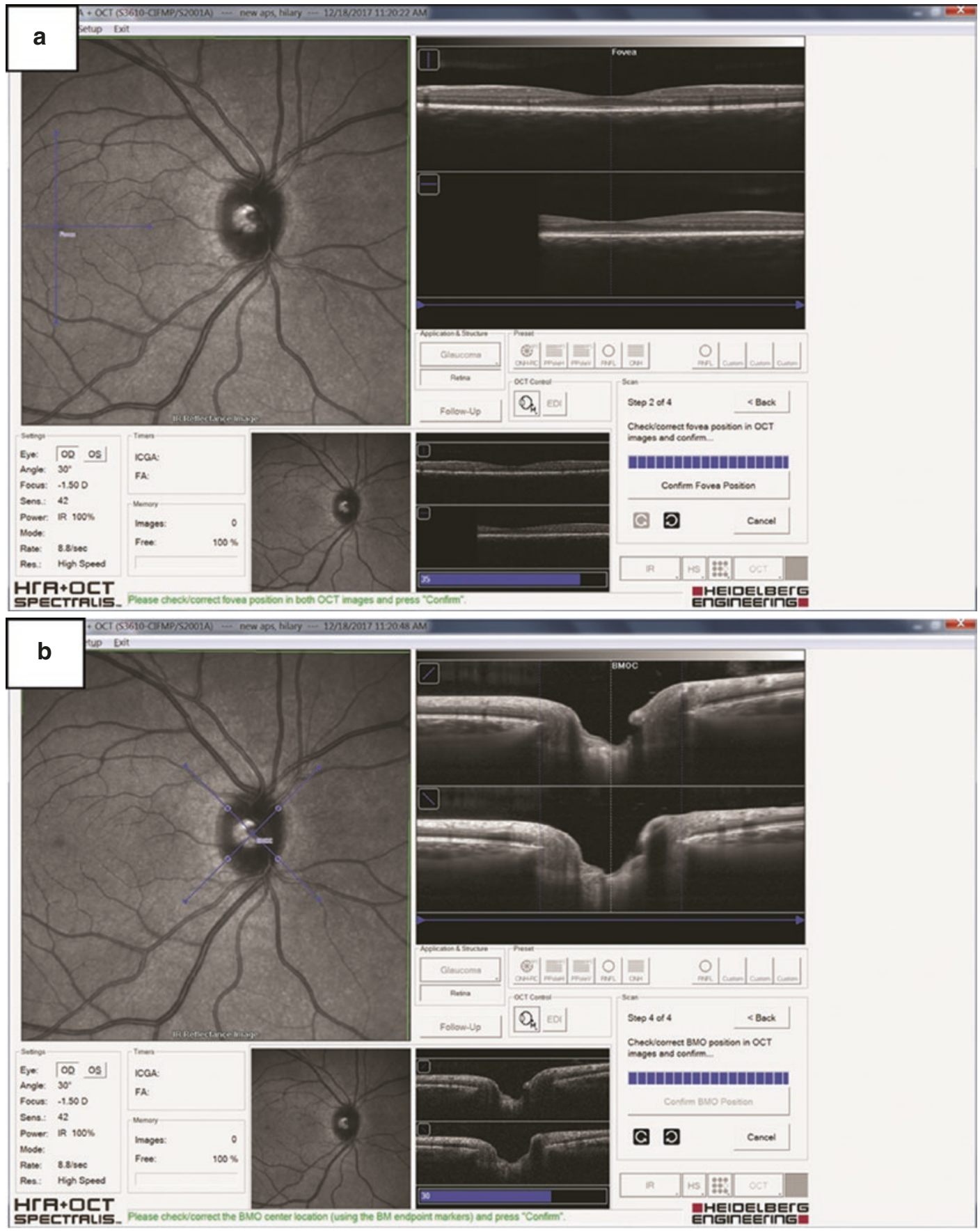

Fig. 5.9 The operator confirms the automated detection of both the fovea (a) and the BMO (b) positioning within the acquisition window. These anatomic positioning sys-

disc margin [88]. Because of this, RNFL thickness is increased in larger optic discs when measured with a fixed scan size [89-91]. These studies indicate that using a fixed scan diameter tem (APS) landmarks serve as the placement points for the ONH-RC scan

without adjusting for ONH size introduces inconsistencies and reduces measurement accuracy. Scaling scan diameter according to the $\mathrm{ONH}$ margin may provide a more accurate diagnostic 
RNFL thickness measurement. For this reason, the SPECTRALIS GMPE results account for the size of the $\mathrm{ONH}$ (defined as BMO area) when comparing each eye's RNFL thickness and BMO-MRW values with the respective reference database.

Visualization and SD-OCT imaging of the ONH are also affected by ocular magnification [92-96]. Magnification is determined by two factors: axial length and corneal power. In eyes with longer axial length, the actual diameter of a fixedsize OCT scan will be larger when it reaches the retinal plane. The cornea provides approximately two-thirds of the eye's total optical power and plays a significant role in determining the scan size on the retina. Without ocular magnification corrections, OCT scans may not correctly scaled, leading to inconsistent measurements of RNFL thickness. The SPECTRALIS GMPE software allows the user to adjust for magnification during acquisition by entering individual corneal curva- ture values and by bringing the optic nerve into sharp focus on the cSLO image (Fig. 5.10).

\subsubsection{Factors that May Confound Measurements and Classifications: Age, Axial length, and Tilted Discs}

There is a negative association between RNFL thickness and age that may explain the higher rate of glaucoma detection in older individuals [97]. The GMPE reference database accounts for this known decline in RNFL thickness due to age. The values collected for the reference database also show a statistically significant negative correlation between age and BMO-MRW, and the software RBD comparisons take into consideration this relationship. The reference database also shows a negative correlation between BMO area and MRW (larger BMO area associated

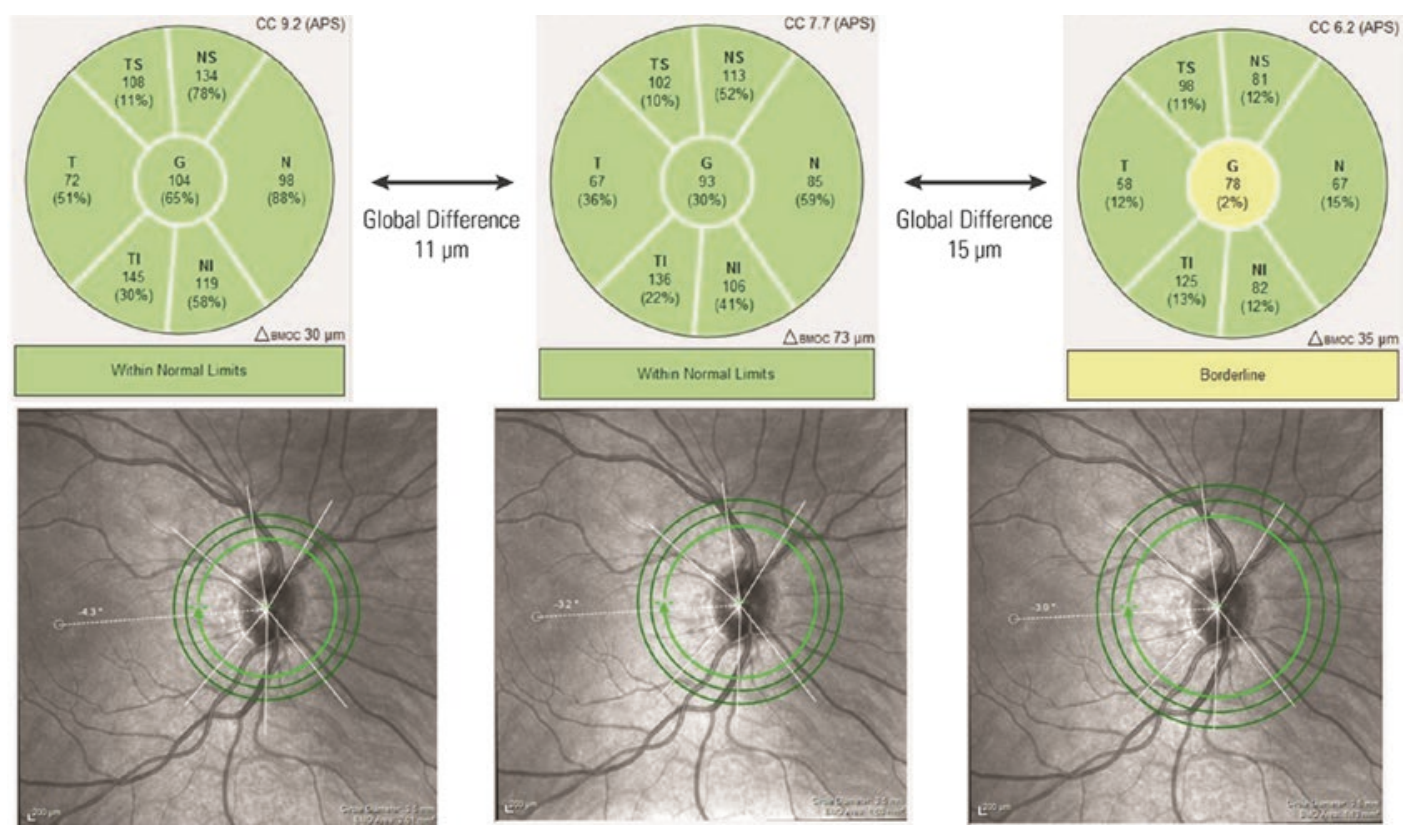

Fig. 5.10 The operator can input the corneal curvature value for each eye so that the fixed-millimeter circle scans are correctly scaled relative to the fundus cSLO image and corresponding $\mathrm{ONH}$ size. This figure shows the range that the circle scans cover across the minimum and maximum corneal curvature values. This eye has a measured corneal curvature (CC) of $7.7 \mathrm{~mm}$. Note that with a high CC value, the circle scans are closer to the disc margin than they should be. With lower CC values, the scans are farther from the disc margin than they should be. Because the RNFL is thinner with increasing distance from the disc margin, the sectorial and global results compared with the reference database are considerably different due to changes in scaling and magnification that are caused by the disparate corneal curvature values 
with thinner MRW) and a positive correlation between BMO area and circumpapillary RNFL thickness (larger BMO area associated with thicker cpRNFL measurements), so the reference database comparisons also are scaled by the $\mathrm{BMO}$ area.

As axial length and spherical equivalent (SE) refractive error increase, the measured average RNFL thickness decreases [98, 99]. This anatomic relationship can decrease the diagnostic power of a reference database, and most devices have a defined range of SE values of -6 to +6 diopters. Even within the included diopter range, the RNFL thickness profile plotted in the circular TSNIT (temporal-superior-inferior-nasal-temporal) profile can be shifted (Fig. 5.11). Considering these anatomical variations, when the SD-OCT reference database metrics do not agree with visual field tests and/or the clinical examination, it may be helpful to review the SD-OCT B-Scans in order to evaluate the overall appearance of the RNFL to help confirm a positive or negative glaucoma diagnosis. The high-quality SPECTRALIS B-Scans offer the detail to evaluate these structures. It may also be useful to consider macular GCL thickness and BMO-MRW measurements in such cases [100, 101].

The tilted disc phenomenon is another confounding anatomical feature that can affect glaucoma diagnosis. Tilted discs can be congenital or, more commonly, can occur in cases of myopia. In these eyes, the axons exit the eye via the $\mathrm{ONH}$ at angles that make assessment of the BMO center and BMO-MRW challenging. In addition, such anatomic anomalies result in arcuate RNFL patterns that are not accounted for in SD-OCT reference databases, making RNFL thickness comparison inconclusive, especially in the temporal region [100, 102]. Also, the distribution of RNFL in eyes with tilted discs is shifted according to the direction of the tilt [103]. Visual field defects also may mimic glaucomatous defects, further confounding the presentation [104]. These characteristics should be considered when applying SD-OCT to the interpretation of RNFL measurements in eyes with tilted discs (Fig. 5.12). A brief inspection of the SPECTRALIS cSLO image and the BMO-MRW radial B-Scans can clearly show BMO-MRW asymmetry and confirm the presence of a tilted disc.

\subsubsection{Posterior Pole: Macular and Asymmetry Analyses}

The density of RGCs is highest in the macula, and the ganglion cell layer (GCL) measured by SD-OCT is thickest surrounding the fovea. Loss of these cell bodies has been shown to be indicative of early glaucomatous damage [105]. Studies have also shown that glaucomatous damage results in characteristic patterns of ganglion cell degeneration in the macula. These patterns of loss present as arcuate patterns that correspond to the arcuate RNFL patterns of loss, confirming that the ganglion cell somas and their respective axons are degenerating. Such observations have led to the concept of "macular vulnerability zones" in the temporal inferior and temporal superior sectors [81]. Therefore, when reviewing OCT diagnostic results, it is important to assess anatomically corresponding ONH, RNFL and macula data in order to detect patterns common to glaucomatous damage.

The superior and inferior GCL are symmetric across the fovea in healthy eyes, and assessment of vertical GCL asymmetry across the fovea may be a sensitive method for detection of early glaucomatous damage [106]. Early damage also may be present asymmetrically between eyes [107, 108]. Macular analysis may be especially important for patients classified as "Glaucoma Suspects" by ophthalmic exam, and a study found that glaucoma suspects with macular thinning were more likely to subsequently present with visual field loss [109].

The GMPE posterior pole horizontal (PPoleH) scan offers macular thickness maps of total retinal thickness as well as the individual GCL, inner plexiform layer (IPL), and macular RNFL thicknesses. The Posterior Pole Asymmetry Analysis (PPAA) is derived from $\mathrm{PPoleH}$ total retinal thickness, and offers a quantitative and illustrative method to assess the asymmetric loss of macular tissues between the superior and inferior macula as well as between eyes (Fig. 5.13). This 

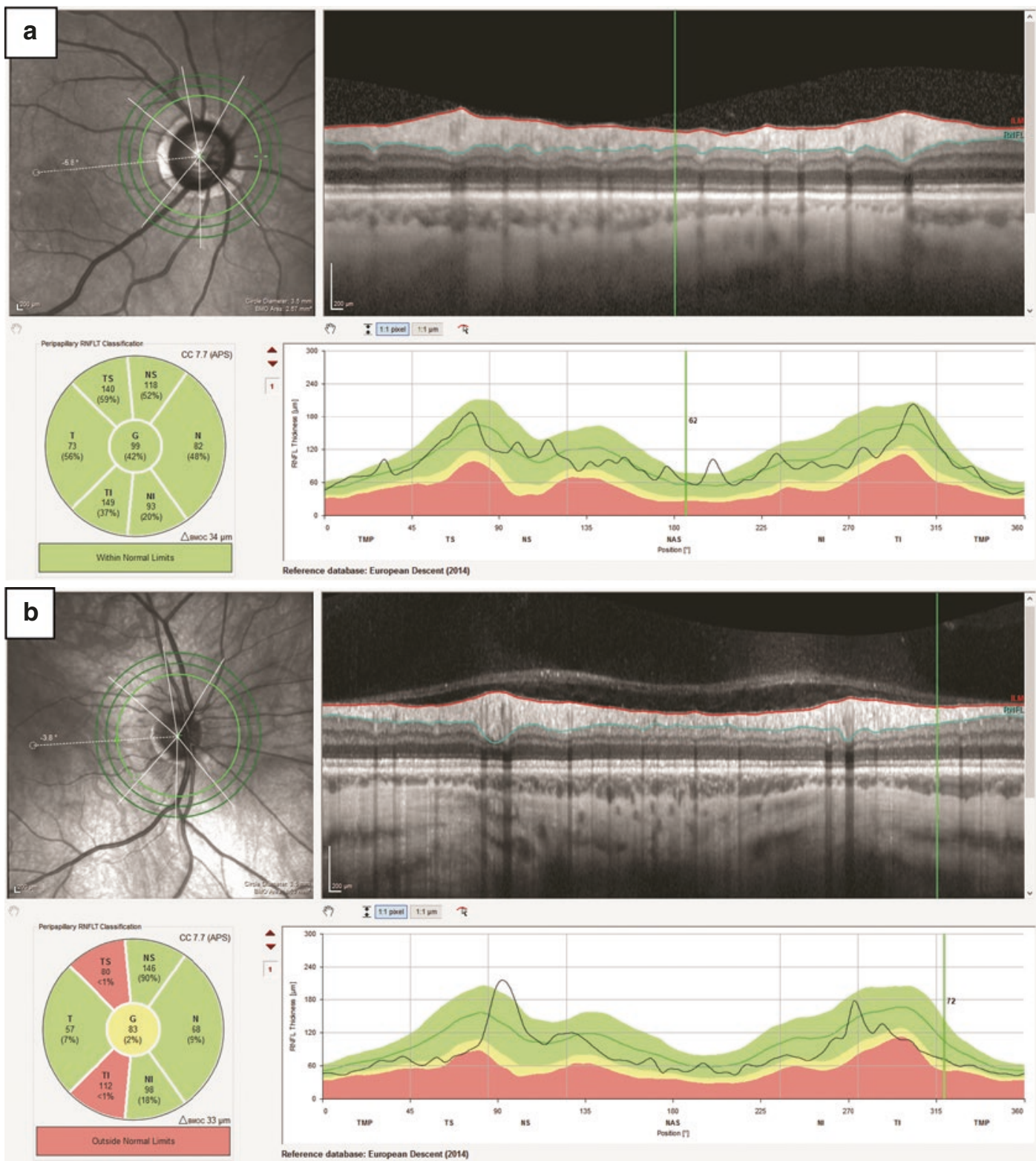

Fig. 5.11 In cases of increased axial length, the RNFL thickness values in the superior and inferior regions may be shifted towards the nasal or temporal sectors. In (a), the normal TSNIT profile has peaks that correspond to the age and BMO-adjusted reference database mean (green shading, solid green line). In a case of myopia (b), the increased axial length causes a shift of the superior and inferior

feature allows clinicians to confirm that the patterns of loss observed on the PPAA and the total retinal thickness maps is in agreement with ganglion cell degeneration patterns that are charac-
RNFL thickness peaks. This causes the 6-sector GarwayHeath analysis to flag the sectors "Outside Normal Limits," but visual inspection of the SD-OCT B-Scans shows a normal, healthy RNFL. Images courtesy Daniel Fuller, OD, Michael Gerstner, OD, and Christopher Lievens, OD, MS

teristic of glaucoma (Fig. 5.14). Regardless of which layers are assessed, macular parameters have been shown to add value as diagnostic tools in the detection of glaucomatous damage [110]. 

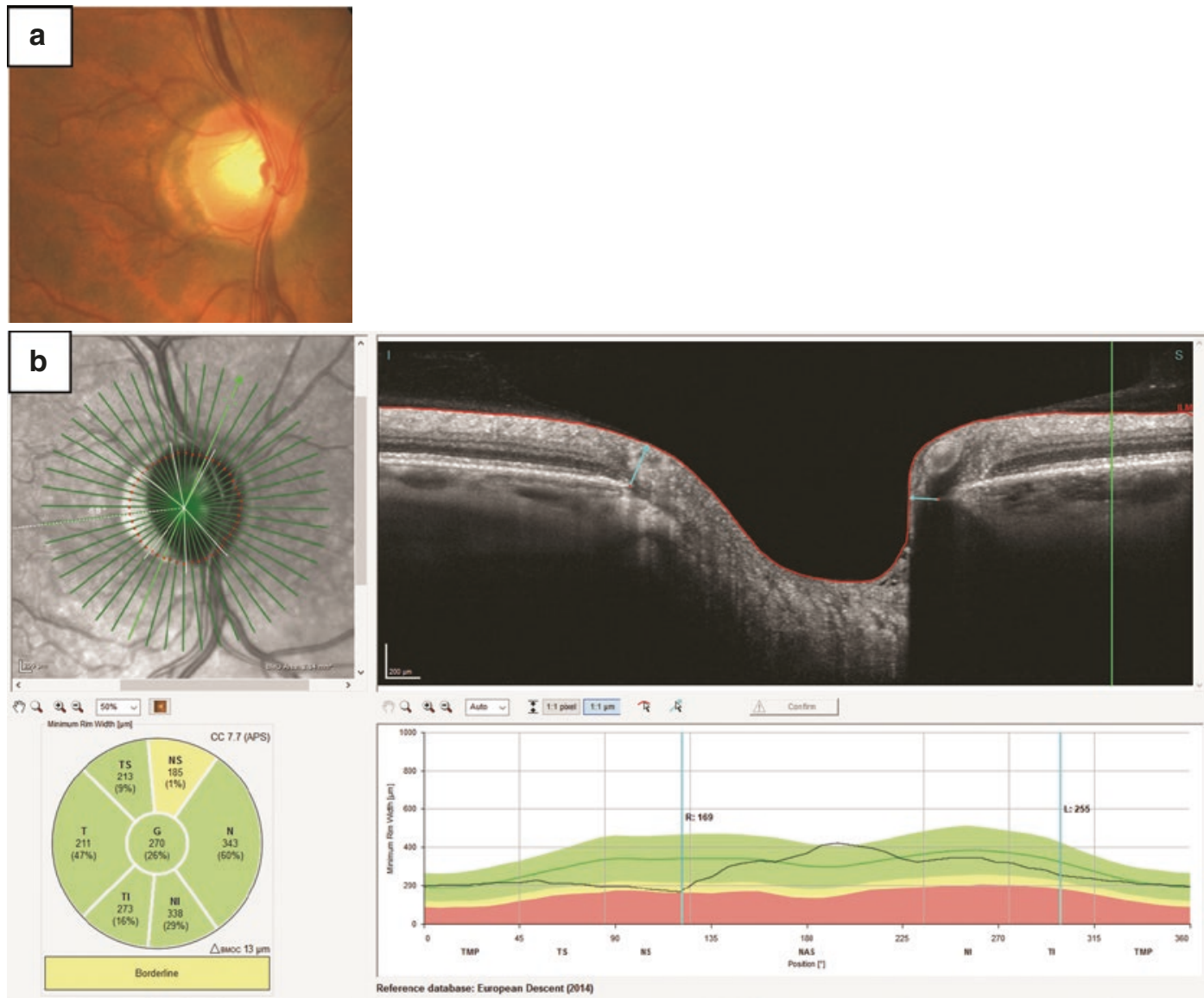

Fig. 5.12 A titled disc can be seen on the fundus photograph (a), and the corresponding BMO endpoints can be identified on the radial scans through the $\mathrm{ONH}(\mathbf{b})$. Similar to cases of increased axial length, there can be shifts in the TSNIT profile of BMO-MRW that cause false-positive comparisons to the reference database. Visual inspection of the SD-OCT B-Scans reveals a healthy neuroretinal rim. Images courtesy Mohammad Rafieetary, OD

In order to accurately assess progression, test measurements need to have adequate reproducibility $[111,112]$. If test measurements have poor reproducibility or are contaminated by noise, detection of true structural loss is not possible. Pierro et al. evaluated retinal nerve fiber layer thickness (RNFLT) measurements using seven different OCTs (spectral- and time-domain), to assess inter- and intra-operator reproducibility of RNFLT [113]. They determined that the SPECTRALIS SD-OCT showed the best reproducibility among the tested devices. The SPECTRALIS eye tracking and ART image acquisition help provide high-quality OCT B-Scans, which in turn allow for reproducible segmentation of the RNFL. In a separate study of 

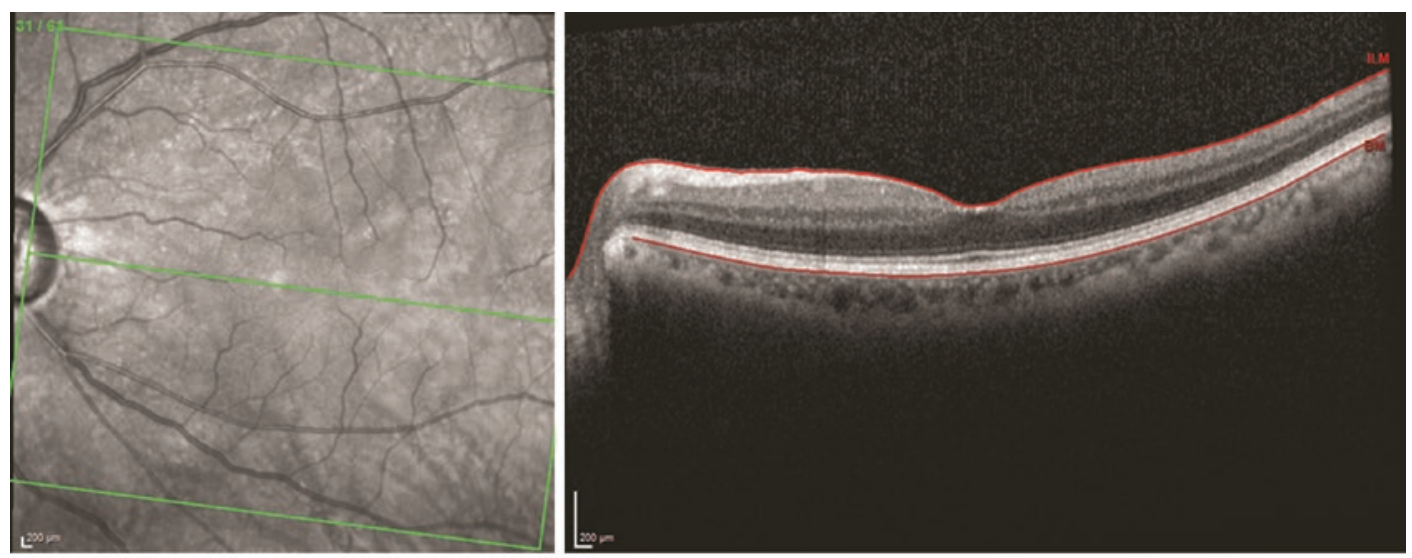

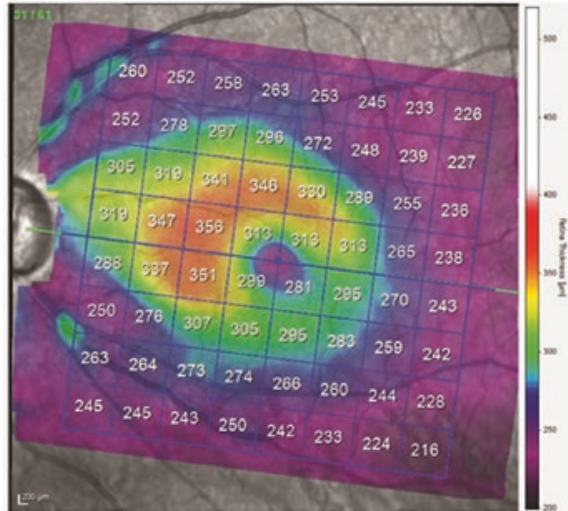

Fig. 5.13 The 61-line posterior pole horizontal scan produces a total retinal thickness color map (lower left). The $8 \times 8$ grid of thickness values serves as the basis for the Posterior Pole Asymmetry Analysis (PPAA) in the lower right panel. The difference in thickness for each corresponding square across the horizontal meridian is shown,

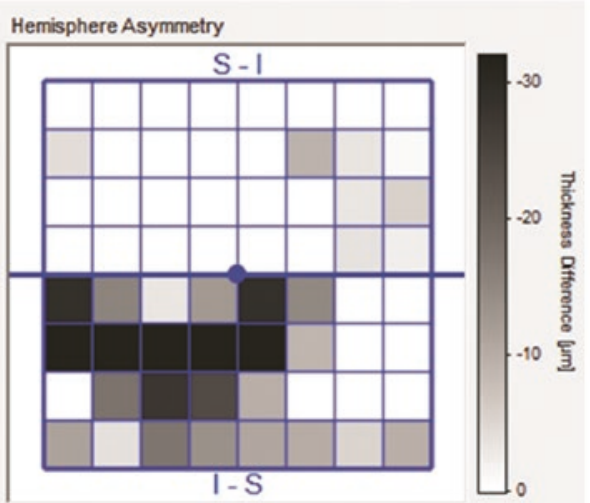

with darker values indicating a larger asymmetric difference in thickness. This analysis can be used to visualize and quantify areas of retinal thickness loss that are characteristic of glaucoma. Images courtesy Shinji Ohkubo, $\mathrm{MD}, \mathrm{PhD}$, and Kazuhisa Sugiyama, MD, $\mathrm{PhD}$

losses from normal aging and beyond the possible noise in repeated measurements.

The ability of a device to measure change over long periods of time also depends on its dynamic range (i.e. the maximum and minimum RNFL thickness measurements that can be reliably made). The lower end of the dynamic range is the "floor" of the measurement, or the minimum layer thickness that is reliably measureable. It is important for an SD-OCT device to have a large dynamic range and a low "floor" value in order to be able to monitor disease progression over time, particularly in advanced glaucoma. The global cpRNFLT dynamic range of the SPECTRALIS has been shown to be larger than comparable devices, while also offering a lower floor [115]. Nevertheless, monitoring advanced glaucoma findings indicate that SD-OCT imaging can be used to detect glaucomatous changes beyond 

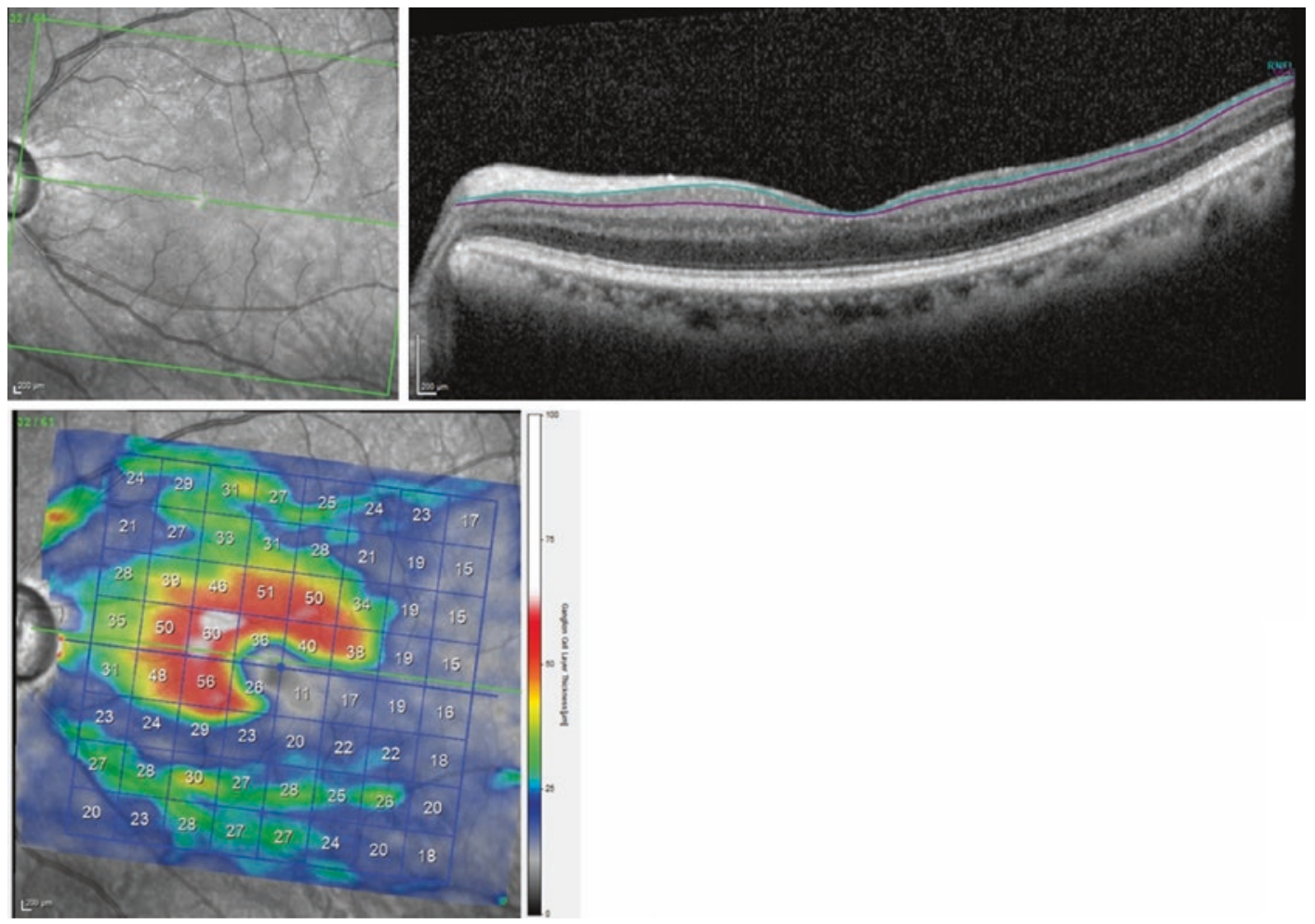

Fig. 5.14 Segmentation of the PPoleH scans between the RNFL and the ganglion cell layer (GCL) provides a GCL thickness color map. In this eye, the same as in Fig. 5.13, there is clear GCL loss in the inferior temporal region

using standard structural and functional testing is extremely difficult for the treating clinician because both standard structural and functional tests that usually guide treatment decisions are of diminished value. Standard structural measures have a limited dynamic range and visual field (VF) test points are more variable in advanced disease [81, 115-120]. However, Belghith et al. have shown that even in very advanced glaucoma, structural loss can be detected in some eyes using standard global structural measures with macular GCIPL identifying the highest proportion of eyes with detectable change, followed by MRW and cpRNFL [121]. In a subsequent study, Bowd et al. concluded that in advanced glaucoma, more macular tissue remains above the measurement floor compared with other measurements, suggesting that macular thickness is the better candidate for detecting progression of such eyes and that progression with SD-OCT measurements is observable in advanced disease [122-125].

\subsubsection{Summary}

Because every eye is unique, it is also important for imaging technologies to incorporate analysis tools that allow for the detection of change in a specific eye over time. The SPECTRALIS SD-OCT provides follow-up scans that are coregistered to baseline imaging, which improves repeatability and makes measurement more precise. The combination of high reproducibility, large dynamic range, a low measurement floor and multiple diagnostic parameters such as the BMO-MRW, cpRNFL and macular thickness measurements allows the device to offer a precise and clinically sensitive progression analysis for all stages of the disease. 
Several studies have investigated the diagnostic performance of various SD-OCT parameters in a standard ophthalmic environment, and the literature suggests that circumpapillary RNFL thickness, $\mathrm{ONH}$, and macular parameters are specific and sensitive for the detection of glaucoma [107, 126-128]. The SPECTRALIS GMPE software offers multiple measurements of ocular structures that, taken together, can offer confirmatory information and increase confidence in the diagnosis. It has been shown that multiple parameters, when used effectively and with caution, are better than any single parameter for diagnosis and management of glaucoma [129]. More importantly, there is clear utility in looking beyond reports at the individual OCT scans, as these can help clarify and reconcile outlying and aberrant outputs.

\subsection{Summary and Outlook}

The development and clinical implementation of cSLO technology (Heidelberg Retina Tomograph) was one of the first imaging technologies in ophthalmology that introduced the ability to objectively quantify various diagnostic parameters for the assessment and management of glaucoma. The technology's diagnostic parameters offered clinicians an objective and precise method to aid their decision in the diagnosis and management of the disease. While the clinical implementation of the HRT served to improve patient care, several clinical research studies employed SLO technology to derive potential surrogate endpoints in clinical trials.

SD-OCT is a more recent technology which also provides objective and quantitative methods to assess the optic nerve head as well as the retinal nerve fiber layer and macula. A current version of this technology, the SPECTRALIS SD-OCT with OCT2 Module, provides a new level of high-resolution imaging of $\mathrm{ONH}$ anatomic features that are affected in glaucoma. This device provides clinicians with the ability to visualize optic nerve structures such as the anterior and in some eyes posterior lamina cribrosa surfaces, Bruch's membrane-retinal pigment epi- thelium complex and its termination within the ONH border, tissue of Elschnig, choroid, and the scleral canal opening.

The ability to visualize the relevant ocular structures in three dimensions in-vivo for the diagnosis and management of glaucoma and to carefully implement multiple objective parameters enables clinicians to make more confident diagnostic decisions. Structural assessment using the imaging technologies discussed in this chapter provides reproducible quantitative measurements of posterior segment ocular structures relevant to the disease.

A newer development in OCT technology, OCT Angiography (OCTA), has sparked interest in evaluating vascular alterations in the retina and $\mathrm{ONH}$ for diagnosis, staging, and monitoring in glaucoma. OCTA is an extension of OCT which allows non-invasive visualization of the retinal vasculature by detecting signal changes induced within perfused blood vessels without the use of exogenous dye. In principle, OCTA compares sequential B-Scans acquired at the same location to detect change. As stationary structures would appear static in sequential B-Scans, changes detected by OCTA are largely attributed to erythrocyte movement in the perfused vasculatures.

Because OCTA offers the non-invasive assessment of microvasculature in the peripapillary retina and macula, it is being investigated for its potential to assess ocular hemodynamics in various diseases [130]. Several OCTA studies have shown reduced microcirculation in the peripapillary retina and the superficial macula of openangle glaucoma eyes, with a moderate relationship between microvasculature and function [131-133].

An early OCTA study suggested that the OCTA vessel density parameter may identify glaucomatous damage before focal visual field defects are detectable [132]. Another study showed that macular vessel density results correlate with central 10-2 visual field defects [133]. The same group also concluded that macular and peripapillary vascular density measurements detected changes in retinal microvasculature before visual field damage in the unaffected eyes of glaucoma patients presenting with unilateral 
glaucoma [134]. Moghimi et al. showed that lower baseline OCTA parameters were associated with a faster rate of RNFL progression in mild to moderate glaucoma over a mean follow-up of 27 months, suggesting that decreased vessel density may be a risk indicator for progression [135].

It remains controversial whether OCTA measurements have a higher diagnostic performance for glaucoma detection compared to conventional OCT measurements such as RNFL thickness, neuroretinal rim width, and macular ganglion cell and inner plexiform layer thickness. Chen and colleagues demonstrated that OCTA-measured vessel density and circumpapillary RNFL thickness measurements had comparable diagnostic performance for the detection of glaucoma suspect and glaucoma eyes [136]. Another recent study compared the diagnostic performance for glaucoma detection between OCTA vessel density measurements (defined using a non-commercially available method) and structural OCT RNFL thickness measurements. Results indicated that vessel area density measurements had a significantly smaller area under the receiver operating characteristic curve for classifying healthy versus glaucoma eyes than RNFL thickness measurements [137], in disagreement with several similar studies. However, classification performance of healthy and glaucoma suspect eyes were similar for vessel area density and RNFL thickness measurements. There is also evidence that deep-layer microvasculature dropout of the parapapillary choriocapillaris or microvasculature within the sclera occurs in eyes with glaucoma and is associated with more severe visual field damage and occurs more frequently in eyes with disc hemorrhages and RNFL thinning [63, 138-140].

While findings from the literature comparing OCTA and structural OCT measurements for the detection of glaucoma and evaluation of structurefunction association are divergent, the potential ability to elucidate the temporal sequence of vascular changes and optic nerve damage in glaucoma may pave the way for better understating and management of the disease.

During the past three decades, advancements in ophthalmic diagnostic imaging technologies have enabled the ability to detect glaucomatous neuropathy at early stages of disease. These advanced imaging technologies provide large amounts of reproducible data, allowing clinicians to discriminate between normal and glaucomatous optic nerves in a more systematic way. From the earlier stages of cSLO imaging to the more recent implementations of OCT technology, several decades of technical and clinical work have been united and integrated in a high-technology solution to give physicians the ability to look deep in the pathological process of glaucoma and to develop better diagnostics and therapeutic strategies. The development of ophthalmic diagnostic imaging technology and the continued efforts to enhance it continues to benefit patients that suffer from glaucoma.

Gerhard Zinser, $\mathrm{PhD}$ was an inspiration to us all. He was a visionary in ophthalmic imaging and an innovative scientist who pursued collaborations and supported new ideas from investigators and clinicians from all over the world. He recognized the importance of designing instruments that produce the best quality scans possible, while also making sure users understood the strengths and limitations of the technology. I have strong memories of sitting for hours with Gerhard and our reading center team in San Diego reviewing images, developing and refining quality control criteria and discussing strategies for analysis to ensure that the highest quality information would be available for the Diagnostic Innovations in Glaucoma Study (DIGS), the African Descent and Glaucoma Evaluation Study (ADAGES) and the Ocular Hypertension Treatment Study (OHTS). Our research was accelerated through his support of promising research in our laboratory, and numerous discussions about how to translate the results into clinical care. His passing is a great loss to the vision science community. (Linda Zangwill, $\mathrm{PhD}$ )

Acknowledgement Supported in part by National Institutes of Health/National Eye Institute grants R01 EY029058 (RNW), R01 EY027510 (LZ), R01 EY011008, R01 EY014267, Core Grant P30EY022589, and by an unrestricted grant from Research to Prevent Blindness (New York, NY).

\section{References}

1. Lichter PR. Variability of expert observers in evaluating the optic disc. Trans Am Ophthalmol Soc. 1976;74:532-72.

2. Gaasterland DE, Blackwell B, Dally LG, et al. The Advanced Glaucoma Intervention Study (AGIS): 10. Variability among academic glaucoma subspe- 
cialists in assessing optic disc notching. Trans Am Ophthalmol Soc. 2001;99:177-84.

3. Jampel HD, Friedman D, Quigley H, et al. Agreement among glaucoma specialists in assessing progressive disc changes from photographs in open-angle glaucoma patients. Am J Ophthalmol. 2009;147(1):39-44.e1.

4. Burnstein Y, Ellish NJ, Magbalon M, Higginbotham EJ. Comparison of frequency doubling perimetry with Humphrey visual field analysis in a glaucoma practice. Am J Ophthalmol. 2000;129(3):328-33.

5. Sekhar GC, Naduvilath TJ, Lakkai M, et al. Sensitivity of Swedish interactive threshold algorithm compared with standard full threshold algorithm in Humphrey visual field testing. Ophthalmology. 2000;107(7):1303-8.

6. Wadood AC, Azuara-Blanco A, Aspinall P, et al. Sensitivity and specificity of frequency-doubling technology, tendency-oriented perimetry, and Humphrey Swedish interactive threshold algorithmfast perimetry in a glaucoma practice. Am J Ophthalmol. 2002;133(3):327-32.

7. Johnson CA, Sample PA, Cioffi GA, et al. Structure and function evaluation (SAFE). I. Criteria for glaucomatous visual field loss using standard automated perimetry (SAP) and short wavelength automated perimetry (SWAP). Am J Ophthalmol. 2002;134(2):177-85.

8. Keltner JL, Johnson CA, Quigg JM, et al. Confirmation of visual field abnormalities in the Ocular Hypertension Treatment Study. Ocular Hypertension Treatment Study Group. Arch Ophthalmol. 2000;118(9):1187-94.

9. Bowd C, Zangwill LM, Medeiros FA, et al. Confocal scanning laser ophthalmoscopy classifiers and stereophotograph evaluation for prediction of visual field abnormalities in glaucoma-suspect eyes. Invest Ophthalmol Vis Sci. 2004;45:2255-62.

10. Miglior S, Zeyen T, Pfeiffer N, Cunha-Vaz J, Torri V, Adamsons I. Results of the European glaucoma prevention study. Ophthalmology. 2005;112:366-75.

11. Sommer A, Katz J, Quigley HA, et al. Clinical detectable nerve fiber atrophy precedes the onset of glaucomatous field loss. Arch Ophthalmol. 1991;109:77-83.

12. Zeyen TG, Caprioli J. Progression of disc and field damage in early glaucoma. Arch Ophthalmol. 1993;111:62-5.

13. Sommer A, Miller NR, Pollack I, Maumenee AE, George T. The nerve fiber layer in the diagnosis of glaucoma. Arch Ophthalmol. 1977;95:2149-56.

14. Kass MA, Heuer DK, Higginbotham EJ, et al. The ocular hypertension treatment study - a randomized trial determines that topical ocular hypotensive medication delays or prevents the onset of primary open-angle glaucoma. Arch Ophthalmol. 2002;120(6):701-13.

15. Zangwill LM, Weinreb RN, Beiser JA, et al. Confocal Scanning Laser Ophthalmoscopy Ancillary Study to the Ocular Hypertension Treatment Study
Group. Baseline topographic optic disc measurements are associated with the development of primary open-angle glaucoma. Arch Ophthalmol. 2005;123:1188-97.

16. Miki A, Medeiros FA, Weinreb RN, Jain S, He F, Sharpsten L, Khachatryan N, Hammel N, Liebmann JM, Girkin CA, Sample PA, Zangwill LM. Rates of retinal nerve fiber layer thinning in glaucoma suspect eyes. Ophthalmology. 2014;121:1350-8.

17. Kuang TM, Zhang C, Zangwill LM, Weinreb RN, Medeiros FA. Estimating lead time gained by optical coherence tomography in detecting glaucoma before development of visual field defects. Ophthalmology. 2015; $122: 2002$. ophtha.2015.06.015.

18. Sommer A, Miller NR, Pollack I, et al. The nerve fiber layer in the diagnosis of glaucoma. Arch Ophtalmol. 1977;95:2149-56.

19. Leske MC, Heijl A, Hussein M, et al. Factors for glaucoma progression and the effect of treatment: the early manifest glaucoma trial. Arch Ophthalmol. 2003;121(1):48-56.

20. Goldberg I. Relationship between intraocular pressure and preservation of visual field in glaucoma. Surv Ophthalmol. 2003;48:S3-7.

21. The Advanced Glaucoma Intervention Study (AGIS); The AGIS Investigators. 7. The relationship between control of intraocular pressure and visual field deterioration. Am J Ophthalmol. 2000;130(4):429-40.

22. Hattenhauer MG, Johnson DH, Ing HH, et al. The probability of blindness from open-angle glaucoma. Ophthalmology. 1998;105(11):2099-104.

23. Chen PP. Blindness in patients with treated openangle glaucoma. Ophthalmology. 2003;110(4):726.

24. Oliver JE, Hattenhauer MG, Herman D, et al. Blindness and glaucoma: a comparison of patients progressing to blindness from glaucoma with patients maintaining vision. Am J Ophthalmol. 2002;133(6):764-72.

25. Fayers T, Strouthidis NG, Garway-Heath D. Monitoring glaucomatous progression using a novel Heidelberg Retina Tomograph event analysis. Ophthalmology. 2007;114:1973-80.

26. Artes P, Chauhan B. Longitudinal changes in the visual field and optic disc in glaucoma. Prog Retin Eye Res. 2005;24:333-54.

27. Chauhan BC, Blanchard JW, Hamilton DC, LeBlanc RP. Technique for detecting serial topographic changes in the optic disc and peripapillary retina using scanning laser tomography. Invest Ophthalmol Vis Sci. 2000;41:775-82.

28. Weinreb RN, Lusky M, Bartsch DU, Morsman D. Effect of repetitive imaging on topographic measurements of the optic nerve head. Arch Ophthalmol. 1993;111(5):636-8.

29. Dreher AW, Tso PC, Weinreb RN. Reproducibility of topographic measurements of the normal and glaucomatous optic nerve head with the laser tomographic scanner. Am J Ophthalmol. 1991;111(2):221-9. 
30. Lusky M, Bosem ME, Weinreb RN. Reproducibility of optic nerve head topography measurements in eyes with undilated pupils. J Glaucoma. 1993;2(2):104-9.

31. Zangwill L, Shakiba S, Caprioli J, Weinreb RN. Agreement between clinician and a confocal scanning laser ophthalmoscope in estimating cup/ disk ratios. Am J Ophthalmol. 1995;119(4):415-21.

32. Anton A, Yamagishi N, Zangwill L, et al. Mapping structural to functional damage in glaucoma with standard automated perimetry and confocal scanning laser ophthalmoscopy. Am J Ophthalmol. 1998;125(4):436-46.

33. Wollstein G, Garway-Heath DF, Hitchings RA. Identification of early glaucoma cases with the scanning laser ophthalmoscope. Ophthalmology. 1998;105(8):1557-63.

34. Swindale NV, Stjepanovic G, Chin A, et al. Automated analysis of normal and glaucomatous optic nerve head topography images. Invest Ophthalmol Vis Sci. 2000;41(7):1730-42.

35. Tipping ME. Sparse Bayesian learning and the relevance vector machine. J Mach Learn Res. 2001;1:211-44.

36. Chauhan BC, Blanchard JW, Hamilton DC, et al. Technique for detecting serial topographic changes in the optic disc and peripapillary retina using scanning laser tomography. Invest Ophthalmol Vis Sci. 2000;41(3):775-82.

37. Bowd C, Balasubramanian M, Weinreb RN, et al. Performance of confocal scanning laser tomograph Topographic Change Analysis (TCA) for assessing glaucomatous progression. Invest Ophthalmol Vis Sci. 2009;50(2):691-701.

38. Gordon MO, Kass MA. The ocular hypertension treatment study: design and baseline description of the participants. Arch Ophthalmol. 1999;117(5):573-83.

39. Zangwill LM, Weinreb RN, Berry CC, et al. The confocal scanning laser ophthalmoscopy ancillary study to the ocular hypertension treatment study: study design and baseline factors. Am J Ophthalmol. 2004;137(2):219-27.

40. Zangwill LM, Weinreb RN, Berry CC, et al. Racial differences in optic disc topography: baseline results from the confocal scanning laser ophthalmoscopy ancillary study to the ocular hypertension treatment study. Arch Ophthalmol. 2004;122:22-8.

41. Weinreb RN, Zangwill LM, Jain S, et al. Predicting the onset of glaucoma: the confocal scanning laser ophthalmoscopy ancillary study to the Ocular Hypertension Treatment Study. Ophthalmology. 2010;117(9):1674-83.

42. Alencar LM, Bowd C, Weinreb RN, et al. Comparison of HRT-3 glaucoma probability score and subjective stereophotograph assessment for prediction of progression in glaucoma. Invest Ophthalmol Vis Sci. 2008;49(5):1898-906.

43. Chauhan BC, Nicolela MT, Artes PH. Incidence and rates of visual field progression after longitu- dinally measured optic disc change in glaucoma. Ophthalmology. 2009;116(11):2110-8.

44. Medeiros FA, Lisboa R, Zangwill LM, et al. Evaluation of progressive neuroretinal rim loss as a surrogate end point for development of visual field loss in glaucoma. Ophthalmology. 2014;121(1):100-9.

45. Sample PA, Girkin CA, Zangwill LM, Jain S, Racette L, Becerra LM, Weinreb RN, Medeiros FA, Wilson MR, De Leon-Ortega J, Bowd C. Liebmann JM for the ADAGES Study Group. The African descent and glaucoma evaluation study (ADAGES): design and baseline data. Arch Ophthalmol. 2009;127:1136.

46. Girkin CA, Sample PA, Liebmann JM, Jain S, Bowd C, Becerra LM, Medeiros FA, Racette L, Dirkes KA, Weinreb RN, Zangwill LM. ADAGES Study Group. African Descent and Glaucoma Evaluation Study (ADAGES): 2. Ancestry differences in optic disc, retinal nerve fiber layer, and macular structure in normal subjects. Arch Ophthalmol. 2010;128:541-50.

47. Medeiros FA, Zangwill LM, Bowd C, et al. Agreement between stereophotographic and confocal scanning laser ophthalmoscopy measurements of cup/disc ratio: effect on a predictive model for glaucoma development. J Glaucoma. 2007;16:209-14.

48. Medeiros FA, Alencar LM, Zangwill LM, et al. Prediction of functional loss in glaucoma from progressive optic disc damage. Arch Ophthalmol. 2009;127(10):1250-6.

49. Zangwill LM, Jain S, Dirkes K, et al. Confocal scanning laser ophthalmoscopy ancillary study to the ocular hypertension treatment study. Am J Ophthalmol. 2013;155(6):971-82.

50. Hammel N, Belghith A, Bowd C, et al. Rate and pattern of rim area loss in healthy and progressing glaucoma eyes. Ophthalmology. 2016;123:760-70.

51. Leite MT, Rao HL, Zangwill LM, Weinreb RN, Medeiros FA. Comparison of the diagnostic accuracies of the Spectralis, Cirrus, and RTVue optical coherence tomography devices in glaucoma. Ophthalmology. 2011;118:1334.

52. Silverman AL, Hammel N, Khachatryan N, Sharpsten L, Medeiros FA, Girkin CA, Liebmann JM, Weinreb RN, Zangwill LM. Diagnostic accuracy of the spectrails and cirrus reference databases in differentiating between healthy and early glaucoma eyes. Ophthalmology. 2016;123(2):408-14.

53. Armaly MF. Lessons to be learned from a glaucoma survey. J Iowa State Med Soc. 1960;50:501-7.

54. Balazsi AG, Drance SM, Schulzer M, Douglas GR. Neuroretinal rim area in suspected glaucoma and early chronic open-angle glaucoma: correlation with parameters of visual function. Arch Ophthalmol. 1984;102:1011-4.

55. Reis AS, Sharpe GP, Yang H, et al. Optic disc margin anatomy in patients with glaucoma and normal controls with spectral domain optical coherence tomography. Ophthalmology. 2012;119:738-47.

56. Strouthidis NG, Yang H, Reynaud JF, et al. Comparison of clinical and spectral domain optical 
coherence tomography optic disc margin anatomy. Invest Ophthalmol Vis Sci. 2009;50(10):4709-18.

57. Agoumi Y, Sharpe GP, Hutchison DM, et al. Laminar and prelaminar tissue displacement during intraocular pressure elevation in glaucoma patients and healthy controls. Ophthalmology. 2011;118(1):52-9.

58. Strouthidis NG, Grimm J, Williams GA, et al. A comparison of optic nerve head morphology viewed by spectral domain optical coherence tomography and by serial histology. Invest Ophthalmol Vis Sci. 2010;51(3):1464-74.

59. Srinivasan VJ, Adler DC, Chen Y, et al. Ultrahighspeed optical coherence tomography for threedimensional and en face imaging of the retina and optic nerve head. Invest Ophthalmol Vis Sci. 2008;49(11):5103-10.

60. Lee EJ, Kim TW, Weinreb RN, et al. Visualization of the lamina cribrosa using enhanced depth imaging spectral-domain optical coherence tomography. Am J Ophthalmol. 2011;152:87-95.

61. Park HY, Jeon SH, Park CK. Enhanced depth imaging detects lamina cribrosa thickness differences in normal tension glaucoma and primary open-angle glaucoma. Ophthalmology. 2012;119(1):10-20.

62. Loureiro MM, Vianna JR, Danthurebandara VM, et al. Visibility of optic nerve head structures with spectral-domain and swept-source optical coherence tomography. J Glaucoma. 2017;26(9):792-7.

63. Suh MH, Zangwill LM, Manalastas PIC, et al. Deeplayer microvasculature dropout by optical coherence tomography angiography and microstructure of parapapillary atrophy. Invest Ophthalmol Vis Sci. 2018;59(5):1995-2004.

64. Manalastas PIC, Belghith A, Weinreb RN, et al. Automated beta zone parapapillary area measurement to differentiate between healthy and glaucoma eyes. Am J Ophthalmol. 2018;191:140-8.

65. Belghith A, Bowd C, Medeiros FA, Weinreb RN, Zangwill LM. Automated segmentation of anterior lamina cribrosa surface: how the lamina cribrosa responds to intraocular pressure change in glaucoma eyes. IEEE International Symposium on Biomedical Imaging ISBI, New York. Washington, DC: IEEE; 2015. p. 222-5.

66. Reis AS, O'Leary N, Yang H, et al. Influence of clinically invisible, but optical coherence tomography detected, optic disc margin anatomy on neuroretinal rim evaluation. Invest Ophthalmol Vis Sci. 2012;53:1852-60.

67. Chen TC. Spectral domain optical coherence tomography in glaucoma: qualitative and quantitative analysis of the optic nerve head and retinal nerve fiber layer (An AOS Thesis). Trans Am Ophthalmol Soc. 2009;107:254-81.

68. Povazay B, Hofer B, Hermann B, et al. Minimum distance mapping using three-dimensional optical coherence tomography for glaucoma diagnosis. J Biomed Opt. 2007;12(4):041204.

69. Belghith A, Bowd C, Medeiros FA, et al. Does the location of bruch's membrane opening change over time? Longitudinal analysis using san diego automated layer segmentation algorithm (SALSA). Invest Ophthalmol Vis Sci. 2016;57:675-82.

70. Ramrattan RS, van der Schaft TL, Mooy CM, et al. Morphometric analysis of Bruch's membrane, the choriocapillaris, and the choroid in aging. Invest Ophthalmol Vis Sci. 1994;35:2857-64.

71. Hu Z, Abràmoff MD, Kwon YH, Lee K, Garvin MK. Automated segmentation of neural canal opening and optic cup in 3D spectral optical coherence tomography volumes of the optic nerve head. Invest Ophthalmol Vis Sci. 2010;51(11):5708-17.

72. Reis AS, O'Leary N, Stanfield MJ, et al. Laminar displacement and prelaminar tissue thickness change after glaucoma surgery imaged with optical coherence tomography. Invest Ophthalmol Vis Sci. 2012;53(9):5819-26.

73. Jonas JB, Gusek GC, Guggenmoos-Holzmann I, Naumann GO. Correlations of the neuroretinal rim area with ocular and general parameters in normal eyes. Ophthalmic Res. 1988;20(5):298-303.

74. Strouthidis NG, Fortune B, Yang H, Sigal IA, Burgoyne CF. Longitudinal change detected by spectral domain optical coherence tomography in the optic nerve head and peripapillary retina in experimental glaucoma. Invest Ophthalmol Vis Sci. 2011;52(3):1206-19.

75. Chauhan BC, O'Leary N, AlMobarak FA, et al. Enhanced detection of open-angle glaucoma with an anatomically accurate optical coherence tomography-derived neuroretinal rim parameter. Ophthalmology. 2013;120:535-43.

76. Toshev AP, Lamparter J, Pfeiffer N, Hoffmann EM. Bruch's membrane opening-minimum rim width assessment with spectral-domain optical coherence tomography performs better than confocal scanning laser ophthalmoscopy in discriminating early glaucoma patients from control subjects. J Glaucoma. 2017;26(1):27-33.

77. Gmeiner JM, Schrems WA, Mardin CY, et al. Comparison of Bruch's membrane opening minimum rim width and peripapillary retinal nerve fiber layer thickness in early glaucoma assessment. Invest Ophthalmol Vis Sci. 2016;57:OCT575-84.

78. Ghassibi MP, Chien JL, Patthanathamrongkasem $\mathrm{T}$, et al. Glaucoma diagnostic capability of circumpapillary retinal nerve fiber layer thickness in circle scans with different diameters. J Glaucoma. 2017;26(4):335-42.

79. Jonas RA, Wang YX, Yang H, et al. Optic disc fovea angle: the Beijing eye study 2011. PLoS One. 2015;10:e1041771.

80. Airaksinen P, Tuulonen A, Werner E. Clinical evaluation of the optic disc and retinal nerve fiber layer. In: Ritch R, Shields M, Krupin T, editors. The glaucomas. St. Louis, MO: Mosby; 1996. p. 617-57.

81. Hood DC, Raza AS, de Moraes CG, Liebmann JM, Ritch R. Glaucomatous damage of the macula. Prog Retin Eye Res. 2013;32:1-21. 
82. Patel NB, Wheat JL, Rodriguez A, Tran V, Harwerth RS. Agreement between retinal nerve fiber layer measures from Spectralis and Cirrus spectral domain OCT. Optom Vis Sci. 2012;89(5):E652-66.

83. Caprioli J, Miller JM. Correlation of structure and function in glaucoma. Quantitative measurements of disc and field. Ophthalmology. 1988;95(6):723-7.

84. Hood DC, Anderson SC, Wall M, Raza AS, Kardon RH. A test of a linear model of glaucomatous structure-function loss reveals sources of variability in retinal nerve fiber and visual field measurements. Invest Ophthalmol Vis Sci. 2009;50(9):4254-66.

85. Bin Ismail MA, Hui Li Lilian K, Yap SC, Yip LW. Effect of head tilt and ocular compensatory mechanisms on retinal nerve fiber layer measurements by cirrus spectral domain and spectralis optical coherence tomography in normal subjects. J Glaucoma. 2016;25:579-83.

86. Schuman JS, Pedut-Kloizman T, Hertzmark E, et al. Reproducibility of nerve fiber layer thickness measurements using optical coherence tomography. Ophthalmology. 1996;103:1889-98.

87. Jonas JB, Budde WM, Panda-Jonas S. Ophthalmoscopic evaluation of the optic nerve head. Surv Ophthalmol. 1999;43(4):293-320.

88. Varma R, Skaf M, Barron E. Retinal nerve fiber layer thickness in normal human eyes. Ophthalmology. 1997;103:2114-9.

89. Savini G, Zanini M, Carelli V, et al. Correlation between retinal nerve fibre layer thickness and optic nerve head size: an optical coherence tomography study. Br J Ophthalmol. 2005;89(4):489-92.

90. Skaf M, Bernardes AB, Cardillo JA, et al. Retinal nerve fibre layer thickness profile in normal eyes using third-generation optical coherence tomography. Eye (Lond). 2006;20(4):431-9.

91. Nagai-Kusuhara A, Nakamura M, Fujioka M, Tatsumi Y, Negi A. Association of retinal nerve fibre layer thickness measured by confocal scanning laser ophthalmoscopy and optical coherence tomography with disc size and axial length. Br J Ophthalmol. 2008;92(2):186-90.

92. Bengtsson B, Krakau C. Correction of optic disc measurements on fundus photographs. Graefes Arch Clin Exp Ophthalmol. 1992;230:24-8.

93. Garway-Heath DF, Rudnicka AR, Lowe T, et al. Measurement of optic disc size: equivalence of methods to correct for ocular magnification. $\mathrm{Br} \mathrm{J}$ Ophthalmol. 1998;82:643-9.

94. Bengtsson B. The variation and covariation of cup and disc diameters. Acta Ophthalmol. 1972;54:804-18.

95. Budenz DL, Anderson DR, Varma R, et al. Determinants of normal retinal nerve fiber layer thickness measured by Stratus OCT. Ophthalmology. 2007;114(6):1046-52.

96. Wakitani Y, Sasoh M, Sugimoto M, et al. Macular thickness measurements in healthy subjects with different axial lengths using optical coherence tomography. Retina. 2003;23:177-82.
97. Alasil T, Wang K, Keane PA, et al. Analysis of normal retinal nerve fiber layer thickness by age, sex, and race using spectral domain optical coherence tomography. J Glaucoma. 2013;22(7):532-41.

98. Wang G, Qiu KL, Lu XH, et al. The effect of myopia on retinal nerve fibre layer measurement: a comparative study of spectral-domain optical coherence tomography and scanning laser polarimetry. $\mathrm{Br} \mathrm{J}$ Ophthalmol. 2011;95(2):255-60.

99. Seo S, Lee CE, Jeong JH, et al. Ganglion cell-inner plexiform layer and retinal nerve fiber layer thickness according to myopia and optic disc area: a quantitative and three-dimensional analysis. BMC Ophthalmol. 2017;17(1):22.

100. Lee K, Kim C, Kim N. Variations of retinal nerve fiber layer thickness and ganglion cell-inner plexiform layer thickness according to the torsion direction of optic disc. Invest Ophthalmol Vis Sci. 2014;55:1048-55.

101. Rebolleda G, Casado A, Oblanca N, Muñoz-Negrete FJ. The new Bruch's membrane opening - minimum rim width classification improves optical coherence tomography specificity in tilted discs. Clin Ophthalmol. 2016;10:2417-25.

102. Hwang Y, Yoo C, Kim Y. Myopic optic disc tilt and the characteristics of peripapillary retinal nerve fiber layer thickness measured by spectral-domain optical coherence tomography. J Glaucoma. 2012;21:260-5.

103. Law SK, Tamboli DA, Giaconi J, Caprioli J. Characterization of retinal nerve fiber layer in nonglaucomatous eyes with tilted discs. Arch Ophthalmol. 2010;128:141-2.

104. Vuori M, Mäntyjärvi M. Tilted disc syndrome may mimic false visual field deterioration. Acta Ophthalmol. 2008;86:622-5.

105. Nakano N, Hangai M, Nakanishi H, et al. Macular ganglion cell layer imaging in preperimetric glaucoma with speckle noise-reduced spectral domain optical coherence tomography. Ophthalmology. 2011;118(12):2414-26.

106. Yamada H, Hangai M, Nakano N, et al. Asymmetry analysis of macular inner retinal layers for glaucoma diagnosis. Am J Ophthalmol. 2014;158:1318-29.

107. Seo JH, Kim TW, Weinreb RN, et al. Detection of localized retinal nerve fiber layer defects with posterior pole asymmetry analysis of spectral domain optical coherence tomography. Invest Ophthalmol Vis Sci. 2012;53:4347-53.

108. Asrani S, Rosdahl J, Allingham R. Novel software strategy for glaucoma diagnosis: asymmetry analysis of retinal thickness. Arch Ophthalmol. 2011;129(9):1205.

109. Gupta D, Asrani S. Macular thickness analysis for glaucoma diagnosis and management. Taiwan $\mathrm{J}$ Ophthalmol. 2016;6(1):3-7.

110. Chien JL, Ghassibi MP, Patthanathamrongkasem $\mathrm{T}$, et al. Glaucoma diagnostic capability of global and regional measurements of isolated ganglion cell layer and inner plexiform layer. J Glaucoma. 2017;26(3):208-15. 
111. Cremasco F, Massa G, Gonçalves Vidotti V, et al. Intrasession, intersession, and interexaminer variabilities of retinal nerve fiber layer measurements with spectral-domain OCT. Eur J Ophthalmol. 2011;21:264-70.

112. Araie M. Test-retest variability in structural parameters measured with glaucoma imaging devices. Jpn J Ophthalmol. 2013;57:1-24.

113. Pierro L, Gagliardi M, Iuliano L, Ambrosi A, Bandello F. Retinal nerve fiber layer thickness reproducibility using seven different OCT instruments. Invest Ophthalmol Vis Sci. 2012;53:5912-20.

114. Wessel J, Horn F, Tornow R, Schmid M, Mardin $\mathrm{C}$, Kruse F, et al. Longitudinal analysis of progression in glaucoma using spectral-domain optical coherence tomography. Invest Ophthalmol Vis Sci. 2013;54:3613-20.

115. Mwanza JC, Kim HY, Budenz DL, et al. Residual and dynamic range of retinal nerve fiber layer thickness in glaucoma: comparison of three OCT platforms. Invest Ophthalmol Vis Sci. 2015;56:6344-51.

116. Lee JM, Cirineo N, Ramanathan M, et al. Performance of the visual field index in glaucoma patients with moderately advanced visual field loss. Am J Ophthalmol. 2014;157:39-43.

117. Gardiner SK, Swanson WH, Goren D, Mansberger SL, Demirel S. Assessment of the reliability of standard automated perimetry in regions of glaucomatous damage. Ophthalmology. 2014;121:1359-69.

118. Hood DC, Kardon RH. A framework for comparing structural and functional measures of glaucomatous damage. Prog Retin Eye Res. 2007;26:688-710.

119. Mwanza J-C, Budenz DL, Warren JL, et al. Retinal nerve fibre layer thickness floor and corresponding functional loss in glaucoma. Br J Ophthalmol. 2015;99:732-7.

120. Saunders LJ, Medeiros FA, Weinreb RN, Zangwill LM. What rates of glaucoma progression are clinically significant? Exp Rev Ophthalmol. 2016;11:227-34.

121. Belghith A, Medeiros FA, Bowd C, et al. Structural change can be detected in advanced-glaucoma eyes. Invest Ophthalmol Vis Sci. 2016;57(9):OCT511-8.

122. Bowd C, Zangwill LM, Weinreb RN, et al. Estimating optical coherence tomography structural measurement floors to improve detection of progression in advanced glaucoma. Am J Ophthalmol. 2017;175:37-44.

123. Sung KR, Sun JH, Na JH, Lee JY, Lee Y. Progression detection capability of macular thickness in advanced glaucomatous eyes. Ophthalmology. 2012;119:308-13.

124. Shin JW, Sung KR, Lee GC, Durbin MK, Cheng D. Ganglion cell-inner plexiform layer change detected by optical coherence tomography indicates progression in advanced glaucoma. Ophthalmology. 2017;124:1466-74.

125. Sung MS, Kang BW, Kim HG, et al. Clinical validity of macular ganglion cell complex by spectral domain-optical coherence tomography in advanced glaucoma. J Glaucoma. 2014;23:341-6.

126. Lisboa R, Paranhos A Jr, Weinreb RN, et al. Comparison of different spectral domain OCT scanning protocols for diagnosing preperimetric glaucoma. Invest Ophthalmol Vis Sci. 2013;54(5):3417-25.

127. Mwanza JC, et al. Ability of cirrus HD-OCT optic nerve head parameters to discriminate normal from glaucomatous eyes. Ophthalmology. 2011;118(2):241-8.e1.

128. Nukada $\mathbf{M}$, et al. Imaging of localized retinal nerve fiber layer defects in preperimetric glaucoma using spectral-domain optical coherence tomography. J Glaucoma. 2014;23:150-9.

129. Mwanza JC, Oakley JD, Budenz DL, et al. Diagnostic performance of optical coherence tomography ganglion celleinner plexiform layer thickness measurements in early glaucoma. Ophthalmology. 2014;121:849-54.

130. Jia Y, Morrison JC, Tokayer J, et al. Quantitative OCT angiography of optic nerve head blood flow. Biomed Opt Express. 2012;3(12):3127-37.

131. Yarmohammadi A, Zangwill LM, Diniz-Filho A, et al. Optical coherence tomography angiography vessel density in healthy, glaucoma suspect, and glaucoma eyes. Invest Ophthalmol Vis Sci. 2016;57(9):OCT451-9.

132. Yarmohammadi A, Zangwill LM, Diniz-Filho A, et al. Peripapillary and macular vessel density in patients with glaucoma and single-hemifield visual field defect. Ophthalmology. 2017;124(5):709-19.

133. Penteado RC, Zangwill LM, Daga FB, et al. Optical coherence tomography angiography macular vascular density measurements and the central 10-2 visual field in glaucoma. J Glaucoma. 2018;27(6):481-9.

134. Yarmohammadi A, Zangwill LM, Manalastas PIC, et al. Peripapillary and macular vessel density in patients with primary open-angle glaucoma and unilateral visual field loss. Ophthalmology. 2018;125(4):578-87.

135. Moghimi S, Zangwill LM, Penteado RC, et al. Macular and optic nerve head vessel density and progressive retinal nerve fiber layer loss in glaucoma. Ophthalmology. 2018;125(11):1720-8.

136. Chen CL, Zhang A, Bojikian KD, et al. Peripapillary retinal nerve fiber layer vascular microcirculation in glaucoma using optical coherence tomographybased microangiography. Invest Ophthalmol Vis Sci. 2016;57(9):OCT475-85.

137. Rao HL, Pradhan ZS, Weinreb RN, et al. A comparison of the diagnostic ability of vessel density and structural measurements of optical coherence tomography in primary open angle glaucoma. PLoS One. 2017;12(3):e0173930.

138. Suh MH, Zangwill LM, Manalastas PIC, et al. Deep retinal layer microvasculature dropout detected by the optical coherence tomography angiography in glaucoma. Ophthalmology. 2016;123(12):2509-18. 
139. Suh MH, Park JW, Kim HR. Association between the deep-layer microvasculature dropout and the visual field damage in glaucoma. J Glaucoma. 2018;27(6):543-51.
140. Park HL, Kim JW, Park CK. Choroidal microvasculature dropout is associated with progressive retinal nerve fiber layer thinning in glaucoma with disc hemorrhage. Ophthalmology. 2018;125(7):1003-13.

Open Access This chapter is licensed under the terms of the Creative Commons Attribution 4.0 International License (http://creativecommons.org/licenses/by/4.0/), which permits use, sharing, adaptation, distribution and reproduction in any medium or format, as long as you give appropriate credit to the original author(s) and the source, provide a link to the Creative Commons license and indicate if changes were made.

The images or other third party material in this chapter are included in the chapter's Creative Commons license, unless indicated otherwise in a credit line to the material. If material is not included in the chapter's Creative Commons license and your intended use is not permitted by statutory regulation or exceeds the permitted use, you will need to obtain permission directly from the copyright holder. 